\title{
The Weibel instability in relativistic plasmas
}

\section{Linear theory}

\author{
A. Achterberg and J. Wiersma
} Sterrenkundig Instituut, Universiteit Utrecht, PO Box 80000, 3508 TA Utrecht, The Netherlands
e-mail: achterb@astro. uu.nl

Received 5 April 2006 / Accepted 17 July 2007

ABSTRACT

\begin{abstract}
Aims. We discuss the linear theory of the Weibel instability in a relativistic plasma driven by ultra-relativistic beams, describing the physics of the generation of magnetic fields in the ultra-relativistic shocks associated with Gamma Ray Bursts (GRBs). We perform a detailed analysis of the linear dispersion relation for the benefit of non-linear calculations that we discuss in the companion paper. Methods. We use a covariant approach, where the linear response of the beam-plasma system is determined from the polarization tensor. This tensor relates the four-current density to the four-potential of the electromagnetic field. Showing that two approaches, one based on a fluid model and one on a kinetic description that uses a waterbag distribution for the phase-space density of the beam particles, yield essentially the same result, we compare our results to those obtained by other approaches. We mainly consider the symmetric case of two counterstreaming (but otherwise identical) beams.

Results. We show that the effect of an asymmetry in the beam densities is small for typical parameters, and briefly discuss the effect of an ambient magnetic field. The dispersion relation of the Weibel instability driven by ultra-relativistic beams is rather insensitive to the model used to describe the plasma. The properties of the instability, such as the growth rate and the range of unstable wavelengths, are governed by only two parameters: the ratio of the plasma frequency squared of the beam and hot background plasma, and a "Mach number", which is essentially the ratio of the beam momentum and the momentum associated with thermal velocity ( $\sim$ sound speed) in the beam plasma. We also show that, at least for the parameters associated with the ultra-relativistic shocks in GRBs, the influence of the magnetic field is small, and the results for an unmagnetized plasma can be used. Conclusions.
\end{abstract}

Key words. magnetic fields - instabilities - shock waves - gamma rays: bursts

\section{Introduction}

The Weibel instability is an electromagnetic instability of lowfrequency perturbations in a plasma where one (or more) of the particle species has an anisotropic momentum distribution. In its original form, as discussed by Weibel (1959), this anisotropy is caused by different values of the kinetic temperature of the particles in two mutually orthogonal directions. More recent astrophysical applications - see for instance Gruzinov \& Waxman (1999), Gruzinov (2001), Medvedev \& Loeb (1999), and Frederiksen et al. (2004) - have concentrated on the anisotropy caused by the presence of bulk plasma motions in the form of beams in a (thermal) background plasma.

In relativistic shocks, which propagate into a cold medium with bulk speed $V_{\mathrm{sh}} \sim c$, this version of the instability could be important as the collisionless relaxation mechanism in the "transition layer" where the shock-heated and incoming (unshocked) plasma mix. As this instability has its most rapid growth in the low-frequency regime, so that $|\omega| \ll k c$ with $k=2 \pi / \lambda$ the wave number, it generates an electromagnetic field $(\delta \boldsymbol{E}, \delta \boldsymbol{B})$ that is dominated by the magnetic component: $|\delta \boldsymbol{B}| \gg|\delta \boldsymbol{E}|$.

Generation of magnetic fields is a necessary ingredient in those models that explain the prompt gamma rays, and the broadband afterglow emission from GRBs as (Lorentz-boosted) synchrotron radiation. These synchrotron models must assume that the magnetic field is strong in the sense that the ratio of magnetic to internal energy is $B^{2} / 8 \pi e \approx 0.01-0.1$. Here $B$ the magnetic field strength with corresponding energy density $e_{B}=B^{2} / 8 \pi$, and $e$ is the internal energy density of the radiating plasma. Details of the arguments leading to this estimate can be found in the reviews by Piran (2000, 2004) and Mészáros (2002, 2006), and in the references therein.

The compression of pre-existing interstellar (or circumstellar) magnetic fields by a relativistic shock leads to an insufficient field strength, with $\epsilon_{B} \ll 1$. In fact, without additional field amplification one finds that the pre- and post-shock values of $\epsilon_{B} \equiv B^{2} / 8 \pi e$ are of similar magnitude, see Eq. (4) below. For an exterior shock, propagating into a cold astrophysical plasma with proper mass density $\rho$, the upstream energy density is dominated by the rest-energy density of the hadrons: $e \simeq \rho c^{2}$. Therefore, the pre-shock value of the equipartition parameter $\epsilon_{B 1}$ is small:

$\epsilon_{B 1} \approx B_{1}^{2} / 8 \pi \rho_{1} c^{2}=\left(V_{\mathrm{A}}^{2} / 2 c^{2}\right)_{1} \ll 1$.

Here, $V_{\mathrm{A}}=B / \sqrt{4 \pi \rho}$ is the (non-relativistic) Alfvén velocity, and we use the subscript 1 (2) to denote the pre-shock (postshock) values of physical quantities.

In the best possible case, where the pre-shock magnetic field is tangential to the shock surface with strength $B_{\mathrm{t}}$, the shock jump conditions (in particular mass conservation and magnetic flux conservation) imply that the pre- and post shock magnetic fields are related by

$\frac{B_{\mathrm{t} 1}}{\rho_{1}}=\frac{B_{\mathrm{t} 2}}{\rho_{2}}$ 
Since the upstream magnetic field is dynamically unimportant (Eq. (1)), one can apply the approximate hydrodynamic jump conditions of Blandford \& McKee (1976). For an ultrarelativistic shock with $\gamma_{\mathrm{sh}}=1 / \sqrt{1-V_{\mathrm{sh}}^{2} / c^{2}} \gg 1$, propagating into a cold medium with mass density $\rho_{1}$ and pressure $P_{1} \ll \rho_{1} c^{2}$, the relativistically hot post-shock gas has a proper mass density $\rho_{2}$, pressure $P_{2}$, and a thermal energy density $e_{2}$ given by

$\frac{\rho_{2}}{\rho_{1}}=2 \sqrt{2} \gamma_{\mathrm{sh}}, \quad e_{2}=3 P_{2}=2 \gamma_{\mathrm{sh}}^{2} \rho_{1} c^{2}$.

Relations (2) and (3) imply that the post-shock ratio of magnetic and thermal energy densities is

$\epsilon_{B 2} \equiv \frac{B_{\mathrm{t} 2}^{2}}{8 \pi e_{2}} \approx \frac{B_{\mathrm{t} 1}^{2}}{2 \pi \rho_{1} c^{2}} \approx 4 \epsilon_{B 1} \ll 1$.

This shows that the pre- and post shock values have similar magnitudes. Clearly an additional mechanism is needed to generate a post-shock magnetic field with $\epsilon_{B 2} \sim 0.01-0.1$.

This paper is concerned with the linear stage of the Weibel instability, where the electromagnetic field amplitudes grow exponentially, $|\delta \boldsymbol{E}|,|\delta \boldsymbol{B}| \propto \exp (\tilde{\sigma} t)$, with $\tilde{\sigma}=\operatorname{Im}(\omega)$ the growth rate. Starting from first principles, we present a detailed analysis of the dispersion relation that determines the growth rate $\tilde{\sigma}$, both in a fluid description of the plasma and in a kinetic waterbag description. These results are important for the analysis of the non-linear stage of the relativistic Weibel instability, which is sensitive to the dispersion properties of the plasma; see the companion paper (Achterberg et al. 2007) for details. From the dispersion relation we derive expressions for the growth rate $\tilde{\sigma}$ using various approximations: non-relativistic and ultrarelativistic; with and without a background magnetic field. The aim is to determine the basic parameters that characterize the solution.

In Sect. 2 we introduce the covariant formalism employed here and the general form of the plasma response. In Sect. 3 we derive the linear dispersion relation for the beam-driven Weibel instability from two points of view: a fully relativistic fluid plasma version and a kinetic version, both for two counterstreaming beams. In the latter case we employ a mathematically convenient model for the momentum distribution of the beam particles: the waterbag distribution. In Sect. 4 we consider the case of cold relativistic beams and the non-relativistic limit, showing that our results agree with those available for these two cases in the literature. In Sect. 5 we discuss the Weibel instability driven by two symmetric, counterstreaming beams. There we show that the fluid and kinetic results are closely related and are characterized by only two parameters: the ratio of effective plasma frequencies of beams and background plasma and an effective Mach number of the beam plasma. In Sect. 6 we consider the ultra-relativistic limit where the beam velocity $V_{\mathrm{b}}$ is close to $c$ and the beam Lorentz factor satisfies $\gamma_{\mathrm{b}} \gg 1$. In Sect. 7 we consider the effect of anomalous dispersion that occurs when the background plasma is relativistically hot, and in Sect. 8 we discuss the magnetized Weibel instability for beams that are aligned with an ambient magnetic field. There we show that, in the ultra-relativistic case, the influence of this magnetic field is small for typical parameters. In Sect. 9 we briefly discuss the asymmetric case, where the instability is driven by relativistic beams of unequal density. Conclusions are presented in Sect. 10. The Appendices A through D contain the necessary mathematical details.

\section{Covariant formulation of plasma dispersion}

Anticipating our application to relativistic shocks, we use a fully relativistic (covariant) formulation that is valid in any (conveniently chosen) reference frame. This has the advantage that we can decompose the dispersion tensor of the plasma into normal modes based on a set of polarization vectors, postponing considerations of the choice of a specific reference frame as long as possible (see below). Co- and contravariant vector and tensor components are related in the usual fashion, e.g. $\tilde{J}_{\mu}=\eta_{\mu \nu} \tilde{J}^{v}$ and $\alpha_{v}^{\mu}=\eta^{\mu \sigma} \alpha_{\sigma v}$, with $\eta_{\mu v}=\eta^{\mu v} \equiv \operatorname{diag}(1,-1,-1,-1)$ the Minkowski metric tensor of flat space-time, which satisfies $\eta_{v}^{\mu}=\delta_{v}^{\mu}$.

The covariant description employed here (and by others, see the references below) expresses the linear electromagnetic response of the plasma in terms of the wave four-current $\tilde{J}^{\mu}(k)=$ $(\tilde{\rho}, \tilde{\boldsymbol{J}}(k))$ and the wave four-potential $\tilde{A}^{\mu}(k)=(\tilde{\Phi}(k), \tilde{\boldsymbol{A}}(k))$ in the Fourier domain through a tensorial relation of the form (e.g. Melrose 2001)

$4 \pi \tilde{J}^{\mu}(k)=\alpha_{v}^{\mu}(k) \tilde{A}^{v}(k)$.

The polarization tensor $\alpha_{v}^{\mu}(k)$ contains all information about the plasma response. Here, and in what follows, we employ a tilde $(\sim)$ to denote the four-potential and four-current density in the Fourier domain (see definition A.3 in Appendix A), which depend on the wavenumber four-vector $k^{\mu}=(\omega / c, \boldsymbol{k})$. Greek indices are used to denote the components of four-vectors.

The dispersion relation for the linear electromagnetic wave modes in the plasma follows from using (5) in the covariant set of Maxwell equations, Eq. (A.1) of Appendix A. The resulting set of linear relations in the Fourier domain takes the form $D^{\mu v}(k) \tilde{A}_{v}(k)=0$, with $D^{\mu v}(k) \equiv(k \cdot k) \eta^{\mu v}-k^{\mu} k^{v}+\alpha^{\mu v}(k)$, see Appendix B. This leads to three independent physical wave modes. It is convenient to expand the four-potential of the three physical degrees of freedom in terms of three mutually orthogonal polarization vectors: $\tilde{A}^{\mu}(k)=\tilde{A}^{i}(k) e_{i}^{\mu}$, with $i=1,2,3$. The dispersion relation, which is the solution condition for the system of equations, then takes the form $\mathcal{D}(\omega, K)=\operatorname{det}\left(\mathcal{D}_{i j}\right)=0$, where the $3 \times 3$ matrix $\mathcal{D}_{i j}$ is defined in terms of $\alpha_{v}^{\mu}, k^{\mu}$ and the three polarization vectors $e_{i}^{\mu}$ of the linear wave modes, see Appendix B, Eq. (B.11). If one chooses a Lorentz gauge for the electromagnetic fields, $k^{\mu} \tilde{A}_{\mu}=0, \mathcal{D}_{i j}$ takes the form:

$\mathcal{D}_{i j}(k)=(k \cdot k) g_{i j}+\alpha_{i j}(k)$.

Here we employ the notation $A \cdot B \equiv \eta_{\mu \nu} A^{\mu} B^{v}$ for the scalar product of two four-vectors, and introduce the quantities $g_{i j} \equiv$ $\left(e_{i} \cdot e_{j}\right)$, and $\alpha_{i j} \equiv e_{i}^{\mu} \alpha_{\mu v} e_{j}^{v}$. We also adhere to the Einstein summation convention for double indices.

This covariant formulation of the linear electromagnetic response of a plasma is based on the work of Melrose (1973), see also Melrose (2001) and Dewar (1977), and is very convenient for this problem. As it is not commonly used, we provide a full set of definitions together with the necessary mathematical details in Appendices A and B. Appendix C contains full the expressions for the components of the dispersion tensor in the different approaches presented below.

\subsection{Polarization tensor in the fluid approximation}

Many of the properties of the beam-driven Weibel instability can be understood from a simple fluid model. Consider a plasma consisting of several particle species, with charge $q_{\mathrm{s}}$, rest mass $m_{\mathrm{s}}$ and four-velocity $U_{s}^{\mu} \equiv\left(\gamma_{\mathrm{s}}, \gamma_{\mathrm{s}} \boldsymbol{V}_{\mathrm{s}}\right)$ for each particle 
species $\mathrm{s}$. The proper density of species $\mathrm{s}$ is $n_{\mathrm{s}}$, the rest mass density is $\rho_{\mathrm{s}}=n_{\mathrm{s}} m_{\mathrm{s}}$, the thermal energy density is $e_{\mathrm{s}}$ and the pressure is $P_{\mathrm{s}}$. We will use units with $c=1$ so that the Lorentz factor is $\gamma_{\mathrm{s}}=1 / \sqrt{1-\left|\boldsymbol{V}_{\mathrm{s}}\right|^{2}}$. However, we will retain $c$ in some expressions for clarity. For the moment, we will neglect the influence of an ambient magnetic field, in effect treating an unmagnetized plasma. The magnetized case will be considered briefly in Sect. 8.

The calculation of the linear response of each species involves the linearization of the covariant equation of motion for species $\mathrm{s}$ in a charged fluid,

$(\rho+e+P)_{\mathrm{s}}\left(U_{\mathrm{s}} \cdot \nabla\right) U_{\mathrm{s}}^{\mu}=h_{\mathrm{s}}^{\mu v} \nabla_{\nu} P_{\mathrm{s}}+n_{\mathrm{s}} q_{\mathrm{s}} F^{\mu v} U_{\mathrm{s} v}$.

Here $h_{\mathrm{s}}^{\mu \nu} \equiv \eta^{\mu \nu}-U_{\mathrm{s}}^{\mu} U_{\mathrm{s}}^{v}$ is the tensor that projects onto the hyperplane perpendicular to the four-velocity $U_{\mathrm{s}}^{\mu} . F^{\mu \nu}=\nabla^{\mu} A^{v}-\nabla^{v} A^{\mu}$ is the Faraday tensor of the electromagnetic field. Equation of motion (7) is supplemented by the continuity equation and an equation of state,

$\boldsymbol{\nabla}_{\mu}\left(n_{\mathrm{s}} U_{\mathrm{s}}^{\mu}\right)=0, \quad P_{\mathrm{s}} \propto n_{\mathrm{s}}^{\Gamma_{\mathrm{s}}}$.

This procedure leads to a polarization tensor $\alpha_{v}^{\mu}=\sum_{s}\left(\alpha^{\mu}{ }_{v}\right)_{\mathrm{s}}$, where the contribution from species $\mathrm{s}$ takes the form (see Eq. (A.24) in the appendix):

$\left(\alpha^{\mu}{ }_{v}\right)_{\mathrm{s}}=-\tilde{\omega}_{\mathrm{ps}}^{2} \mathcal{P}_{\mathrm{s} \alpha}^{\mu}\left(\delta^{\alpha}{ }_{\beta}-\frac{C_{\mathrm{s}}^{2} k^{\alpha} k_{\beta}}{\left(k \cdot U_{\mathrm{s}}\right)^{2}-K_{\mathrm{s} \perp}^{2} C_{\mathrm{s}}^{2}}\right) \mathcal{P}_{\mathrm{s} v}^{\dagger \beta}$.

The projection tensor $\mathcal{P}_{\mathrm{s} v}^{\mu} \equiv \delta_{v}^{\mu}-U_{\mathrm{s}}^{\mu} k_{v} /\left(k \cdot U_{\mathrm{s}}\right)$ and its transpose appear as a result of charge conservation and the invariance of the current density $\tilde{J}^{\mu}$ under electromagnetic gauge transformations (cf. Dewar 1977), see Appendix A for a full discussion. From here onwards $U_{\mathrm{s}}^{\mu}$ is the unperturbed four-velocity of species $\mathrm{s}$.

The relativistic plasma frequency $\tilde{\omega}_{\mathrm{ps}}$ and the sound speed $C_{\mathrm{s}}$ of species s are respectively defined by:

$\tilde{\omega}_{\mathrm{ps}}^{2} \equiv \frac{4 \pi n_{\mathrm{s}}^{2} q_{\mathrm{s}}^{2}}{(\rho+e+P)_{\mathrm{s}}}, \quad C_{\mathrm{s}}^{2} \equiv \frac{\Gamma_{\mathrm{s}} P_{\mathrm{s}}}{(\rho+e+P)_{\mathrm{s}}}$.

The quantity $K_{\mathrm{s} \perp}^{2}$ is the absolute value of the length of the spacelike four-vector $k_{\mathrm{s} \perp}^{\mu} \equiv h_{\mathrm{s}}^{\mu v} k_{\mu}=k^{\mu}-\left(k \cdot U_{\mathrm{s}}\right) U_{\mathrm{s}}^{\mu}$ :

$K_{\mathrm{s} \perp}^{2} \equiv-k_{\mathrm{s} \perp} \cdot k_{\mathrm{s} \perp}=\left(k \cdot U_{\mathrm{s}}\right)^{2}-k \cdot k$.

Choosing the manifestly covariant Lorentz gauge $k_{\mu} \tilde{A}^{\mu}=0$ and using the resulting properties of the three polarization vectors $e_{1}-e_{3}$ (see Appendix B, Eqs. (B.6)-(B.8)), it follows from expression (9) that the contribution of species s to $\alpha_{i j} \equiv$ $e_{i}^{\mu} \alpha_{\mu \nu} e_{j}^{v}$ is

$\left(\alpha_{i j}\right)_{\mathrm{s}}=-\tilde{\omega}_{\mathrm{ps}}^{2}\left\{g_{i j}+\frac{(k \cdot k)\left(1-C_{\mathrm{s}}^{2}\right) U_{\mathrm{s} i} U_{\mathrm{s} j}}{\left(k \cdot U_{\mathrm{s}}\right)^{2}-K_{\mathrm{s} \perp}^{2} C_{\mathrm{s}}^{2}}\right\}$,

where $U_{\mathrm{s} i} \equiv U_{\mathrm{s}} \cdot e_{i}$ and $g_{i j}=e_{i} \cdot e_{j}$.

\subsection{Polarization tensor in the kinetic description}

Although the fluid approach of the previous section is mathematically easier, a more adequate description of the waves and oscillations in the plasma is given by the kinetic approach. This is especially relevant since we will consider plasmas with a finite temperature in the following sections. We will compare results for the two approaches in Sect. 5.
In the kinetic description, one describes the multi-species plasma using the covariant Vlasov equation for the phase-space distribution function $\mathcal{F}_{\mathrm{s}}\left(x^{\mu}, p^{\mu}\right)$ of each species in the plasma:

$$
\frac{\mathrm{d} \mathcal{F}_{\mathrm{s}}}{\mathrm{d} \tau} \equiv \frac{\mathrm{d} x^{\mu}}{\mathrm{d} \tau} \frac{\partial \mathcal{F}_{\mathrm{s}}}{\partial x^{\mu}}+\frac{q_{\mathrm{s}}}{m_{\mathrm{s}}} F^{\mu v} p_{v} \frac{\partial \mathcal{F}_{\mathrm{s}}}{\partial p^{\mu}}=0,
$$

cf. Eqs. (A.25) and (A.26) of Appendix A. As before, $F^{\mu v}=$ $\nabla^{\mu} A^{v}-\nabla^{v} A^{\mu}$ is the Faraday tensor of the electromagnetic field. The position four vector $x^{\mu}$ and momentum four vector $p^{\mu}$ satisfy $\mathrm{d} x^{\mu} / \mathrm{d} \tau=p_{\mu} / m_{\mathrm{s}}$, with $\tau$ the proper time.

Linearizing the Vlasov equation in the Fourier domain, and calculating the four-current density $\tilde{J}^{\mu}$ resulting from the linear perturbation, one can calculate the polarization tensor $\left(\alpha_{v}^{\mu}\right)_{\mathrm{s}}$ for each species.

As was the case in the fluid description, it is convenient to work with the representation of the polarization tensor $\alpha_{i j}$ in the Lorentz gauge. The contribution to this tensor from species $\mathrm{s}$, $\left(\alpha_{i j}\right)_{\mathrm{s}}$, can be written as (see Appendix C, Eqs. (C.7) and (C.8)):

$$
\left(\alpha_{i j}\right)_{\mathrm{s}}=-\bar{\omega}_{\mathrm{ps}}^{2} \int \frac{\mathrm{d}^{3} \boldsymbol{p}}{\gamma(\boldsymbol{p})} f_{0 \mathrm{~s}}(\boldsymbol{p})\left(g_{i j}+\frac{(k \cdot k) p_{i} p_{j}}{(k \cdot p)^{2}}\right) .
$$

Here $f_{0 \mathrm{~s}}(\boldsymbol{p})$ is the ordinary Vlasov distribution of the particles in three-momentum space, normalized to unity so that $\bar{n}_{\mathrm{s}} f_{0 \mathrm{~s}}(\boldsymbol{p}) \mathrm{d}^{3} \boldsymbol{p}$ is the lab-frame number density of particles with their threemomentum in an infinitesimal momentum space volume $\mathrm{d}^{3} \boldsymbol{p}$ around $\boldsymbol{p}$, with $\bar{n}_{\mathrm{s}}$ the density of species $\mathrm{s}$ in the laboratory frame. The frequency $\bar{\omega}_{\mathrm{ps}}$ is the plasma frequency based on the density of species $s$ in the lab frame:

$\bar{\omega}_{\mathrm{ps}}^{2}=\frac{4 \pi q_{\mathrm{s}}^{2} \bar{n}_{\mathrm{s}}}{m_{\mathrm{s}}}$

As before $g_{i j} \equiv e_{i} \cdot e_{j}$, and we define $p_{i}=p \cdot e_{i}$, with $p^{\mu}=(E, \boldsymbol{p})$ the four-momentum vector so that $\gamma(\boldsymbol{p})=\sqrt{1+|\boldsymbol{p}|^{2} / m^{2} c^{2}}$.

\subsection{The cold plasma limit}

Because cold plasmas are already well-studied in the literature we will check our results in later sections by taking the cold limit. In this section we will give an expression for the polarization tensor in the cold limit.

It is well-known (e.g. Melrose 1980) that the fluid approach and the kinetic approach yield the same answer for a cold plasma. Our expressions for $\alpha_{i j}$ satisfy this simple requirement. The cold plasma assumption corresponds to putting $C_{\mathrm{s}}=0$ in expression (12), and to putting $f_{0 \mathrm{~s}}(\boldsymbol{p})=\delta^{3}\left(\boldsymbol{p}-\boldsymbol{p}_{\mathrm{s}}\right)$ in expression (14). Here $\boldsymbol{p}_{\mathrm{s}} \equiv \gamma_{\mathrm{s}} m_{\mathrm{s}} \boldsymbol{V}_{\mathrm{s}}$ is the bulk momentum of particle species $\mathrm{s}$, where we still allow for relativistic bulk motion with Lorentz factor $\gamma_{\mathrm{s}}=1 / \sqrt{1-V_{\mathrm{s}}^{2}} \gg 1$. In that case one has $\bar{n}_{\mathrm{s}}=\gamma_{\mathrm{s}} n_{\mathrm{s}}$ due to Lorentz-contraction, and the two plasma frequencies defined above are related by:

$\tilde{\omega}_{\mathrm{ps}}^{2}=\frac{\bar{\omega}_{\mathrm{ps}}^{2}}{\gamma_{\mathrm{s}}}=\frac{4 \pi q_{\mathrm{s}}^{2} n_{\mathrm{s}}}{m_{\mathrm{s}}}$.

The cold plasma polarization tensor is then given by

$\left(\alpha_{i j}\right)_{\mathrm{cold}}=-\sum_{\mathrm{s}} \tilde{\omega}_{\mathrm{ps}}^{2}\left(g_{i j}+\frac{(k \cdot k) U_{\mathrm{si}} U_{\mathrm{s} j}}{\left(k \cdot U_{\mathrm{s}}\right)^{2}}\right)$,

where the sum is over all plasma species, and $U_{\mathrm{s}}^{\mu}=p_{\mathrm{s}}^{\mu} / m_{\mathrm{s}}=$ $\gamma_{\mathrm{s}}\left(1, \boldsymbol{V}_{\mathrm{s}}\right)$ is the four-velocity of the bulk motion of species s. 


\section{The beam-driven Weibel instability}

We now consider the case of a plasma with the following components: a (multi-species) "background" plasma, at rest in the lab frame with a bulk four-velocity $U_{\mathrm{s}}^{\mu}=(1,0,0,0)$, and two counterstreaming beams of charged particles moving along the $z$-axis, with an associated four-velocity

$U_{ \pm}^{\mu}=\gamma_{\mathrm{b}}\left(1,0,0, \pm V_{\mathrm{b}}\right)$.

The beam Lorentz factor is $\gamma_{\mathrm{b}}=1 / \sqrt{1-V_{\mathrm{b}}^{2}}$. The beam particles have a rest mass $m_{\mathrm{b}}$ and a charge $q_{\mathrm{b}}$. The two beams have a proper density

$n_{ \pm}=\frac{1 \pm \Delta}{2} n_{\mathrm{b}}$

The parameter $\Delta \leq 1$ measures the asymmetry in the strength of the two counterstreaming beams, with the symmetric case of two equal density beams corresponding to $\Delta=0$. For simplicity we assume that the beams consist of a single particle species, and that the temperature of the two beams, quantified by the sound speed $C_{\mathrm{b}}$ of the beam plasma in the fluid description or by the velocity dispersion in the kinetic description (see below), is identical. The case of beams consisting of more than one particle species is a straightforward generalization of the results presented here.

We limit the discussion to the case of waves with a wave vector $\boldsymbol{k}=K \hat{\boldsymbol{x}}$ perpendicular to the beam direction, corresponding with a wave four-vector $k^{\mu}=(\omega, K, 0,0)$ in the lab frame. This choice implies that, for species belonging to the background plasma, one has $k \cdot U_{\mathrm{s}}=\omega$, and $K_{\mathrm{s} \perp}=|K|$. In this case the three polarization vectors can be chosen as $e_{1}^{\mu}=(K, \omega, 0,0) / \sqrt{|k \cdot k|}$, $e_{2}^{\mu}=(0,0,1,0)$ and $e_{3}^{\mu}=(0,0,0,1)$, see Appendix B. These three vectors all satisfy $k \cdot e_{i}=0$, ensuring that $\tilde{A}^{\mu}(k)=\sum_{i} \tilde{A}^{i}(k) e_{i}^{\mu}$ satisfies the Lorentz gauge.

For this choice of $\boldsymbol{k}$, the only non-zero components of the tensor $\mathcal{D}_{i j}$ as defined in Eq. (6) are: $\mathcal{D}_{11}, \mathcal{D}_{22}, \mathcal{D}_{33}$ and $\mathcal{D}_{13}=$ $\mathcal{D}_{31}^{*}$. The dispersion relation $\mathcal{D}(\omega, K) \equiv \operatorname{det}\left(\mathcal{D}_{i j}\right)=0$ factors into

$\mathcal{D}(\omega, K)=\mathcal{D}_{22}\left(\mathcal{D}_{11} \mathcal{D}_{33}-\left|\mathcal{D}_{31}\right|^{2}\right)=0$.

Here $\left|\mathcal{D}_{31}\right|^{2}=\mathcal{D}_{31} \mathcal{D}_{31}^{*}=\mathcal{D}_{31} \mathcal{D}_{13}$. The explicit expressions for the non-zero components of $\mathcal{D}_{i j}$ are listed in Appendix C.

The solution $\mathcal{D}_{22}=0$ corresponds to a stable, purely electromagnetic mode with $\tilde{\boldsymbol{E}} \perp \boldsymbol{k}$ and $\tilde{\boldsymbol{E}} \perp \boldsymbol{V}_{\mathrm{b}}$ in the lab frame, with $\boldsymbol{V}_{\mathrm{b}}= \pm V_{\mathrm{b}} \hat{z}$ the beam velocity. This mode is unaffected by the bulk motion of the forward and backward beam. It will not be considered further.

The remaining two modes, which follow from $\mathcal{D}_{11} \mathcal{D}_{33}-$ $\left|\mathcal{D}_{31}\right|=0$, are mixed in the sense that the wave electromagnetic field in the rest frame of the background plasma is - in general not purely transverse, with $\tilde{\boldsymbol{E}} \perp \boldsymbol{k}$ in the lab frame, or purely longitudinal, with $\tilde{\boldsymbol{E}} \| \boldsymbol{k}$ in the lab frame. Physically, this is due to the fact that the bulk motion of the beams leads to an "advection current" in addition to the usual "conduction current". The advection current arises as charge density perturbations in the beam plasma are dragged along by the beams, whereas the conduction current is due to the perturbations in the velocity of the beam particles. This couples the transverse (current-driven) and longitudinal (charge-driven) response of the beam plasma. However, in the symmetric case of equal beams, $\Delta=0$, the charge density perturbations of the two beams are opposite and cancel each other, but the associated advection currents are equal due to the the opposite sign of the velocity of the two beams. The charge density cancellation results in $\mathcal{D}_{13}=\mathcal{D}_{31}=0$. In that symmetric case the Weibel instability is purely transverse, with $\tilde{\boldsymbol{E}} \perp \boldsymbol{k}$ and $\tilde{\boldsymbol{E}} \| \boldsymbol{V}_{\mathrm{b}}$ in the lab frame. We will mostly consider this symmetric case, where the Weibel instability is the unstable solution branch of the much simplified dispersion relation

$\mathcal{D}_{33}(k)=K^{2}-\omega^{2}+\sum_{s}\left(\alpha_{33}\right)_{s}=0$.

The asymmetric case with $\mathcal{D}_{31} \neq 0$ will be briefly considered in the next section for the case of cold beams in a cold background plasma, and in Sect. 9 for the hot case.

\section{The limit of a cold beam and the non-relativistic limit}

As a check on our results, we briefly consider the limit of a cold beam and cold background plasma, and the non-relativistic limit, for which results are available in the literature. For ease of comparison we reinstate $c$ in this section. As before we assume $\boldsymbol{k}=K \hat{\boldsymbol{x}}$ and $\boldsymbol{V}_{\mathrm{b}}= \pm V_{\mathrm{b}} \hat{z}$ in the laboratory frame, which is the rest frame of the background plasma. Using (6) and (17), the dispersion relation (20) can be written as:

$$
\begin{aligned}
|k \cdot k| & \left\{\left(1-\sum_{\mathrm{s}} \frac{\tilde{\omega}_{\mathrm{ps}}^{2}}{\omega^{2}}\right)\right. \\
& \times\left(K^{2} c^{2}-\omega^{2}+\sum_{\mathrm{s}} \tilde{\omega}_{\mathrm{ps}}^{2}\left[1-\frac{V_{\mathrm{s}}^{2}}{c^{2}}+\frac{K^{2} V_{\mathrm{s}}^{2}}{\omega^{2}}\right]\right) \\
& \left.+\left(\sum_{\mathrm{s}} \frac{\tilde{\omega}_{\mathrm{ps}}^{2}}{\omega^{2}} K V_{\mathrm{s}}\right)^{2}\right\}=0 .
\end{aligned}
$$

Here the sum extends over all species in the plasma: beams and stationary background. The background plasma has $V_{\mathrm{s}}=0$, and the "cold" plasma frequency is $\tilde{\omega}_{\mathrm{ps}}^{2}=4 \pi q_{\mathrm{s}}^{2} n_{\mathrm{s}} / m_{\mathrm{s}}$ for species $\mathrm{s}$, with the density of the two individual beams given by Eq. (19). This dispersion relation agrees with the more general result of Akhiezer et al. (1975) for cold relativistic beams in the case $\boldsymbol{k} \perp$ $\boldsymbol{V}_{\mathrm{b}}$, and with the dispersion relation of Alexandrov et al. (1984), Chap. 6.3.1., for $\boldsymbol{k} \perp \boldsymbol{V}_{\mathrm{b}}$ and zero magnetic field.

In the non-relativistic limit, where $V_{\mathrm{s}} \ll c$ for all species, the unstable solution branch has $\left|\omega^{2}\right| \ll \sum_{\mathrm{s}} \tilde{\omega}_{\mathrm{ps}}^{2}$. The same holds for weak beams in the relativistic case, i.e. when the plasma frequency associated with the beams satisfies $\tilde{\omega}_{\mathrm{pb}}^{2}=4 \pi q_{\mathrm{b}}^{2} n_{\mathrm{b}} / m_{\mathrm{b}} \ll$ $\sum_{\mathrm{s}} \tilde{\omega}_{\mathrm{ps}}^{2}$. If either of these two inequalities applies one make the approximation $1-\sum_{\mathrm{s}} \tilde{\omega}_{\mathrm{ps}}^{2} / \omega^{2} \approx-\sum_{\mathrm{s}} \tilde{\omega}_{\mathrm{ps}}^{2} / \omega^{2}$, and the dispersion relation (22) reduces to

$K^{2} c^{2}-\omega^{2}+\sum_{\mathrm{s}} \tilde{\omega}_{\mathrm{ps}}^{2}\left(\frac{1}{\gamma_{\mathrm{s}}^{2}}+\frac{K^{2} V_{\mathrm{s}}^{2}}{\omega^{2}}\right)-\frac{K^{2}\left(\sum_{\mathrm{s}} \tilde{\omega}_{\mathrm{ps}}^{2} V_{\mathrm{s}}\right)^{2}}{\omega^{2} \sum_{\mathrm{s}} \tilde{\omega}_{\mathrm{ps}}^{2}}=0$

Here $\gamma_{\mathrm{s}}=1 / \sqrt{1-V_{\mathrm{s}}^{2} / c^{2}}$. In the non-relativistic limit, with $V_{\mathrm{s}} \ll$ $c$ and $\gamma_{\mathrm{s}} \simeq 1$, the unstable branch has $\left|\omega^{2}\right| \ll K^{2} c^{2}$ and the 
solution for the growth rate $\tilde{\sigma}(\omega=i \tilde{\sigma})$ is (cf. Akhiezer et al. 1975, Eq. (6.1.5.10))

$\tilde{\sigma}^{2} \approx \frac{K^{2}}{K^{2} c^{2}+\sum_{\mathrm{s}} \tilde{\omega}_{\mathrm{ps}}^{2}}\left\{\sum_{\mathrm{s}} \omega_{\mathrm{ps}}^{2} V_{\mathrm{s}}^{2}-\frac{\left(\sum_{\mathrm{s}} \tilde{\omega}_{\mathrm{ps}}^{2} V_{\mathrm{s}}\right)^{2}}{\sum_{\mathrm{s}} \tilde{\omega}_{\mathrm{ps}}^{2}}\right\}$.

This non-relativistic dispersion relation remains correct even if one assumes that the magnitude of the velocity of the two beams is not equal. In the symmetric case, where $n_{+}=n_{-}=n_{\mathrm{b}} / 2$ and $V_{ \pm}= \pm V_{\mathrm{b}}$, the term $\sum_{\mathrm{s}} \tilde{\omega}_{\mathrm{ps}}^{2} V_{\mathrm{s}}$ vanishes identically and one has:

$\tilde{\sigma}^{2}=\frac{\tilde{\omega}_{\mathrm{pb}}^{2} K^{2} V_{\mathrm{b}}^{2}}{K^{2} c^{2}+\tilde{\omega}_{\mathrm{bg}}^{2}+\tilde{\omega}_{\mathrm{pb}}^{2}}<\tilde{\omega}_{\mathrm{pb}}^{2}\left(\frac{V_{\mathrm{b}}}{c}\right)^{2}$,

where the maximum growth rate, $\tilde{\sigma}_{\max } \approx \tilde{\omega}_{\mathrm{pb}}\left(V_{\mathrm{b}} / c\right)$, occurs when $K^{2} c^{2} \gg \tilde{\omega}_{\mathrm{bg}}^{2}+\tilde{\omega}_{\mathrm{pb}}^{2}$. Here $\tilde{\omega}_{\mathrm{bg}}^{2} \equiv \sum_{\mathrm{s} \in \mathrm{bg}} 4 \pi q_{\mathrm{s}}^{2} n_{\mathrm{s}} / m_{\mathrm{s}}$ is the plasma frequency associated with the background plasma, and $\tilde{\omega}_{\mathrm{pb}}^{2}=4 \pi q_{\mathrm{b}}^{2} n_{\mathrm{b}} / m_{\mathrm{b}}$ is the plasma frequency associated with the beams. Equation (25) corresponds to the well-known expression for the non-relativistic Weibel instability, which Weibel (1959) derived for an unstable plasma without a background plasma $\left(\tilde{\omega}_{\text {bg }}=0\right)$.

It should be emphasized that the net current in the plasma should vanish,

$\boldsymbol{J}=\sum_{\mathrm{s}} n_{\mathrm{s}} q_{\mathrm{s}} V_{\mathrm{s}} \hat{z}=0$

for a cold plasma. Since $\tilde{\omega}_{\mathrm{ps}}^{2} \propto q_{\mathrm{s}}^{2} n_{\mathrm{s}} / m_{\mathrm{s}}$ this implies that the growth rate reducing term $\propto\left(\sum_{\mathrm{s}} \tilde{\omega}_{\mathrm{ps}}^{2} V_{\mathrm{s}}\right)^{2}$ in relation (24) will vanish if the currents in the plasma are carried by a single species, for instance by electrons in beam(s) and background.

\section{Weibel instability driven by two symmetric beams}

We now consider two counterstreaming beams with equal density and equal temperatures that are propagating through a thermal background plasma at rest. In that case one can use dispersion relation (21).

In the fluid approximation, expression for $\mathcal{D}_{33}$ in the set of relations (C.2) for the components of $\mathcal{D}_{i j}$ leads to dispersion relation

$\omega^{2}=K^{2}+\tilde{\omega}_{\mathrm{bg}}^{2}+\tilde{\omega}_{\mathrm{pb}}^{2}\left(1-\frac{\left(\omega^{2}-K^{2}\right) \tilde{V}_{\mathrm{b}}^{2}}{\omega^{2}-K^{2} \tilde{C}_{\mathrm{b}}^{2}}\right)$.

Here we define the two velocities

$\tilde{C}_{\mathrm{b}}=\frac{C_{\mathrm{b}}}{\gamma_{\mathrm{b}} \sqrt{1-C_{\mathrm{b}}^{2} V_{\mathrm{b}}^{2}}}, \quad \tilde{V}_{\mathrm{b}}=\frac{V_{\mathrm{b}}}{\gamma_{\mathrm{C}_{\mathrm{b}}} \sqrt{1-C_{\mathrm{b}}^{2} V_{\mathrm{b}}^{2}}}$

with $\gamma_{\mathrm{C}_{\mathrm{b}}} \equiv 1 / \sqrt{1-C_{\mathrm{b}}^{2}}$. The background and beam plasma frequencies are given respectively by

$\tilde{\omega}_{\mathrm{bg}}^{2} \equiv \sum_{\mathrm{s} \in \mathrm{bg}} \tilde{\omega}_{\mathrm{ps}}^{2}, \quad \tilde{\omega}_{\mathrm{pb}}^{2} \equiv \frac{4 \pi q_{\mathrm{b}}^{2} n_{\mathrm{b}}}{m_{\mathrm{b}} h_{\mathrm{b}}}$,

with $h_{\mathrm{b}} \equiv 1+(e+P)_{ \pm} / n_{ \pm} m_{\mathrm{b}}$ the beam enthalpy per unit mass, which is identical for the two beams in view of our assumption of equal beam temperatures.
In a kinetic description of the beams we use a mathematically convenient momentum distribution function for describing the kinetic version of the Weibel instability: the waterbag distribution as employed by Yoon \& Davidson (1987) and by Silva et al. (2002). This distribution takes the form

$$
\begin{aligned}
f_{0 \mathrm{~b}}(\boldsymbol{p})= & \frac{\Theta\left(p_{x}+p_{x 0}\right)-\Theta\left(p_{x}-p_{x 0}\right)}{2 p_{x 0}} \delta\left(p_{y}\right) \\
& \times\left[\frac{1+\Delta}{2} \delta\left(p_{z}-p_{z 0}\right)+\frac{1-\Delta}{2} \delta\left(p_{z}+p_{z 0}\right)\right] .
\end{aligned}
$$

Here $\Theta(x)=\frac{1}{2}(1+x /|x|)$ is the Heaviside step-function.

The waterbag distribution describes two beams, counterstreaming along the $z$-axis with a lab-frame density $\bar{n}_{ \pm}=(1 \pm$ $\Delta) \bar{n}_{\mathrm{b}} / 2$, and with a momentum dispersion along the $x$-axis that is described by a "top hat" distribution in the range $-p_{x 0} \leq p_{x} \leq$ $p_{x 0}$. The $y$-component of the momentum vanishes identically. Note that the "beam temperature", as defined by the spread in the momentum component $p_{x}$, is the same for both beams. The waterbag distribution allows an analytical calculation of the plasma response, while preserving the two essential features of more realistic distributions: the bulk drift of beam particles in the beam direction and the beam velocity dispersion along the wave vector due to thermal motions in the beams. As we show below, the final results for a waterbag distribution are close to those obtained with the fluid model, strengthening the validity of this approach.

It has been shown by Milosavljevic et al. (2006) that the dispersion relation for a momentum distribution that is isotropic in the plane perpendicular to the beam (so that $p_{y} \neq 0$, but the distribution only depends on $\sqrt{p_{x}^{2}+p_{y}^{2}}$ and $p_{z}$ ) gives very similar, but algebraically more complicated, results.

For the response of the background plasma we will (for the moment) continue to use the results from the fluid approximation, even though strictly speaking one should use kinetic theory in order to take the large velocity dispersion in the background into account. As discussed in Sect. 7 below, this leads to the anomalous skin effect, cf. Lyubarsky \& Eichler (2006). There we will show that the difference between the two approaches is small. Alternatively, one can use the dispersion functions derived recently by Schlickeiser (2004) for a relativistic Maxwellian distribution.

Using the Eq. (C.12) of Appendix C one finds that the Weibel dispersion relation (21) for a symmetric waterbag distribution with $\Delta=0$ becomes

$\omega^{2}=K^{2}+\tilde{\omega}_{\mathrm{bg}}^{2}+\hat{\omega}_{\mathrm{pb}}^{2}\left(\mathcal{G}_{\mathrm{b}}+\frac{K^{2} V_{z 0}^{2}}{\omega^{2}-K^{2} V_{x 0}^{2}}\right)$.

Here we define the following characteristic beam parameters: the beam plasma frequency $\hat{\omega}_{\mathrm{pb}}$, the Lorentz factor $\gamma_{0}$ and the two velocities $V_{x 0}$ and $V_{z 0}$ :

$\hat{\omega}_{\mathrm{pb}}^{2}=\frac{\bar{\omega}_{\mathrm{pb}}^{2}}{\gamma_{0}}=\frac{4 \pi q_{\mathrm{b}}^{2} \bar{n}_{\mathrm{b}}}{\gamma_{0} m_{\mathrm{b}}}, \quad \gamma_{0}=\sqrt{1+\frac{p_{x 0}^{2}}{m_{\mathrm{b}}^{2} c^{2}}+\frac{p_{z 0}^{2}}{m_{\mathrm{b}}^{2} c^{2}}}$,

$V_{x 0}=\frac{p_{x 0}}{\gamma_{0} m_{\mathrm{b}}}, \quad V_{z 0}=\frac{p_{z 0}}{\gamma_{0} m_{\mathrm{b}}}$.

The function $\mathcal{G}_{\mathrm{b}}\left(p_{x 0}, p_{z 0}\right)$ is given by:

$\mathcal{G}_{\mathrm{b}}\left(p_{x 0}, p_{z 0}\right)=\frac{1}{2 V_{x 0}} \ln \left(\frac{1+V_{x 0}}{1-V_{x 0}}\right)-\frac{p_{z 0}^{2}}{p_{z 0}^{2}+m_{\mathrm{b}}^{2}}$. 
When $V_{x 0} \ll 1$ (so that $\gamma_{0} \simeq \gamma_{\mathrm{b}}$ ) the frequency $\hat{\omega}_{\mathrm{pb}}$ reduces to the beam plasma frequency $\tilde{\omega}_{\mathrm{pb}}$ based on the proper density $n_{\mathrm{b}} \approx \bar{n}_{\mathrm{b}} / \gamma_{\mathrm{b}}$ of the beam particles. The beam contribution to $\mathcal{D}_{33}$ (the last term on the right-hand side of Eq. (31)) has been derived before by Silva et al. (2002).

The two dispersion relations (27) and (31) can both be written as a bi-quadratic equation for $\omega$ of the form

$\omega^{4}-\mathcal{B}(K) \omega^{2}+C(K)=0$,

Such a bi-quadratic equation for $\omega$ occurs often in the theory of the Weibel instability (Weibel 1959; see also Schaefer-Rolffs et al. 2006). The two coefficients $\mathcal{B}(K)$ and $C(K)$ are:

$\mathcal{B}(K)= \begin{cases}\tilde{\omega}_{\mathrm{bg}}^{2}+\tilde{\omega}_{\mathrm{pb}}^{2}\left(1-\tilde{V}_{\mathrm{b}}^{2}\right)+K^{2}\left(1+\tilde{C}_{\mathrm{b}}^{2}\right)(\mathrm{fl}) \\ \tilde{\omega}_{\mathrm{bg}}^{2}+\hat{\omega}_{\mathrm{pb}}^{2} \mathcal{G}_{\mathrm{b}}+K^{2}\left(1+V_{x 0}^{2}\right) \quad(\mathrm{wb}),\end{cases}$

and

$C(K)=\left\{\begin{array}{l}\left(\tilde{\omega}_{\mathrm{bg}}^{2}+K^{2}\right) K^{2} \tilde{C}_{\mathrm{b}}^{2}-\tilde{\omega}_{\mathrm{pb}}^{2} K^{2}\left(\tilde{V}_{\mathrm{b}}^{2}-\tilde{C}_{\mathrm{b}}^{2}\right) \\ \left(\tilde{\omega}_{\mathrm{bg}}^{2}+K^{2}\right) K^{2} V_{x 0}^{2}-\hat{\omega}_{\mathrm{pb}}^{2} K^{2}\left(V_{z 0}^{2}-\mathcal{G}_{\mathrm{b}} V_{x 0}^{2}\right)(\mathrm{wb}) .\end{array}\right.$

Here "fl" and "wb" respectively stand for the fluid and waterbag model. The analogy between these two sets of expressions is clear. The solution for $\omega$,

$\omega_{ \pm}^{2}=\frac{1}{2} \mathcal{B} \pm \frac{1}{2} \sqrt{\mathcal{B}^{2}-4 C}$,

has an unstable branch with $\omega_{-}^{2}<0$, i.e. $\omega_{-}=\mathrm{i} \tilde{\sigma}$ with $\tilde{\sigma}>0$, provided $C(K)<0$. This condition defines a maximum unstable wavenumber $K_{\max }$ so that perturbations with

$K^{2}<K_{\max }^{2}= \begin{cases}\tilde{\omega}_{\mathrm{pb}}^{2}\left(\mathcal{M}^{2}-1\right)-\tilde{\omega}_{\mathrm{bg}}^{2} & (\mathrm{fl}) \\ \hat{\omega}_{\mathrm{pb}}^{2} & \left(\mathcal{M}^{2}-\mathcal{G}_{\mathrm{b}}\right)-\tilde{\omega}_{\mathrm{bg}}^{2}(\mathrm{wb})\end{cases}$

are unstable and will grow. Here we define an effective "Mach number" for the beams by

$\mathcal{M} \equiv\left\{\begin{array}{l}\frac{\tilde{V}_{\mathrm{b}}}{\tilde{C}_{\mathrm{b}}}=\frac{\gamma_{\mathrm{b}} V_{\mathrm{b}}}{\gamma_{\mathrm{C}_{\mathrm{b}}} C_{\mathrm{b}}} \text { (fluid model) } \\ \frac{V_{z 0}}{V_{x 0}}=\frac{p_{z 0}}{p_{x 0}} \quad \text { (waterbag model) }\end{array}\right.$

for the fluid and waterbag model respectively. This shows the stabilizing influence of the thermal motion of beam particles in the direction of the wave vector $\boldsymbol{k}=K \hat{\boldsymbol{x}}$, suppressing the instability at sufficiently large values of $|K|$.

\section{The case of ultra-relativistic beams}

We now concentrate on the ultra-relativistic case, of importance for the Weibel instability in the shock transition layer of shocks with bulk velocity $V_{\mathrm{sh}}$ such that $\gamma_{\mathrm{sh}}=1 / \sqrt{1-V_{\mathrm{sh}}^{2}} \gg 1$. In the frame of the hot (shocked) plasma, the unshocked material forms a relativistic beam. This implies $\gamma_{\mathrm{b}} \sim \gamma_{\mathrm{sh}} \gg 1$ and $\tilde{V}_{\mathrm{b}} \gg \tilde{C}_{\mathrm{b}}$ in the fluid case and $p_{z 0} \gg m_{\mathrm{b}}, p_{z 0} \gg p_{x 0}$ in the waterbag case. It is then possible to describe the properties of the symmetric Weibel instability using two parameters. The first is the "Mach number" $\mathcal{M}$ defined in Eq. (39) that now satisfies ${ }^{1}$ $\mathcal{M}^{2} \sim\left(\gamma_{\mathrm{b}} / \gamma_{\mathrm{Cb}} C_{\mathrm{b}}\right)^{2} \gg 1$. The second parameter is a measure of the strength of the two beams, defined by

$\eta \equiv\left\{\begin{array}{l}\tilde{\omega}_{\mathrm{pb}}^{2} / \tilde{\omega}_{\mathrm{bg}}^{2} \text { (fluid model) } \\ \hat{\omega}_{\mathrm{pb}}^{2} / \tilde{\omega}_{\mathrm{bg}}^{2} \text { (waterbag model). }\end{array}\right.$

${ }^{1}$ For an ideal gas one has $C_{\mathrm{b}}<1 / \sqrt{3}$ and $\gamma_{\mathrm{Cb}} C_{\mathrm{b}}<1 / \sqrt{2}$.
The ultra-relativistic limit of the fluid model has $\tilde{C}_{\mathrm{b}} \simeq 1 / \mathcal{M} \ll 1$, $\tilde{V}_{\mathrm{b}} \simeq V_{\mathrm{b}} \simeq 1$, and in the waterbag model one has $\mathcal{G}_{\mathrm{b}} \ll 1$ and $V_{x 0} \simeq 1 / \mathcal{M} \ll V_{z 0} \approx V_{\mathrm{b}} \simeq 1$. To leading order, the expressions (35) and (36) become identical in both models. In terms of $\eta$ and $\mathcal{M}$ one can write:

$\mathcal{B}(K) \simeq \tilde{\omega}_{\text {bg }}^{2}+K^{2}$

$C(K) \simeq K^{2}\left(\frac{\tilde{\omega}_{\mathrm{bg}}^{2}+K^{2}}{\mathcal{M}^{2}}-\eta \tilde{\omega}_{\mathrm{bg}}^{2}\right)$.

Terms of order $1 / \gamma_{\mathrm{b}}^{2}, \tilde{C}_{\mathrm{b}}^{2}$ or $V_{x 0}^{2}$ have been consistently neglected with respect to unity.

If we define a dimensionless growth rate $\sigma$ and a dimensionless wavenumber $\kappa$ by

$\sigma^{2}=-\omega^{2} / \tilde{\omega}_{\text {bg }}^{2}, \quad \kappa=K / \tilde{\omega}_{\text {bg }}$,

dispersion relation (34) can be written as

$\sigma^{4}+\left(1+\kappa^{2}\right) \sigma^{2}-\frac{\kappa^{2}\left(\kappa_{\max }^{2}-\kappa^{2}\right)}{\mathcal{M}^{2}}=0$.

Here we use that $\omega$ is either real (stable modes) or purely imaginary (unstable modes) with $\omega^{2}<0$ in the latter case. The dimensionless maximum wavenumber $\kappa_{\max }$ (see Eq. (38)) of the unstable modes equals in the ultra-relativistic limit:

$\kappa_{\max } \approx \sqrt{\eta \mathcal{M}^{2}-1}$

The Weibel instability occurs only if $\eta \mathcal{M}^{2}>1$ for $0 \leq|\kappa|<\kappa_{\max }$. The unstable branch has a growth rate

$\sigma^{2}=\sqrt{\left(\frac{1+\kappa^{2}}{2}\right)^{2}+\frac{\kappa^{2}\left(\kappa_{\max }^{2}-\kappa^{2}\right)}{\mathcal{M}^{2}}}-\frac{1+\kappa^{2}}{2}$.

In many cases expression (45) can be simplified further. Provided that $\left(1+\kappa^{2}\right)^{2} \gg 4 \kappa^{2}\left(\kappa_{\max }^{2}-\kappa^{2}\right) / \mathcal{M}^{2}$ one can expand the root in (45). This yields:

$\sigma^{2} \simeq \frac{\kappa^{2}\left(\kappa_{\max }^{2}-\kappa^{2}\right)}{\mathcal{M}^{2}\left(1+\kappa^{2}\right)}$

This approximation corresponds to the neglect of the $\sigma^{4}$ term in dispersion relation (43), and is valid for all unstable wavelengths if the beams are weak in the sense that $\eta \ll 1$. In that case one has $\sigma^{2}<\eta \ll 1$. For $\eta$ close to unity so that $\hat{\omega}_{\mathrm{pb}}, \tilde{\omega}_{\mathrm{pb}} \sim \tilde{\omega}_{\mathrm{bg}}$, the approximated solution (46) is valid everwhere except in the vicinity of $|\kappa|=1$ where $\sigma \approx 1$. For very strong beams with $\eta \gg 1$ one has to use the full solution (45) except when $\sqrt{\eta} \ll$ $|\kappa| \leq \kappa_{\max } \sim \sqrt{\eta} \mathcal{M}$.

Figure 1 gives the growth rate of the Weibel instability in units of the background plasma frequency as a function of the dimensionless wavenumber $\kappa\left(=K c / \tilde{\omega}_{\text {bg }}\right.$ if one uses units with $c \neq 1)$. The curves are labeled by the pair of parameters $(\eta$, $\mathcal{M})$. This figure shows that the dimensionless growth rate $\sigma=$ $\operatorname{Im}(\omega) / \tilde{\omega}_{\text {bg }}$ has the following properties:

- For $\kappa=K / \tilde{\omega}_{\text {bg }} \ll 1$ it grows as $\sigma \propto \kappa$. If the approximated solution (46) applies one has

$\sigma \approx \frac{\kappa \kappa_{\max }}{\mathcal{M}} \approx \sqrt{\eta} \kappa$

The last approximation is valid if $\eta<1$. 


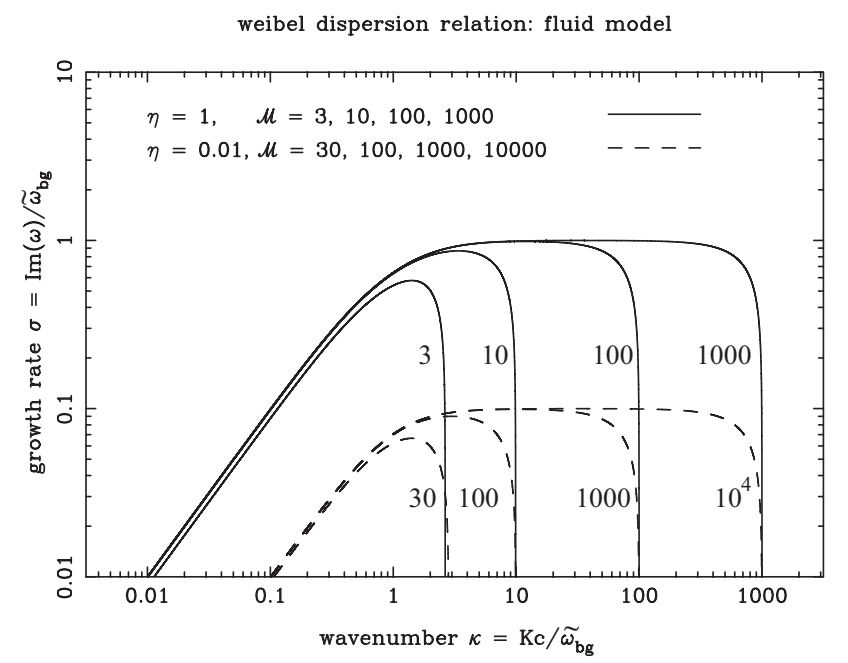

Fig. 1. The dimensionless growth rate of the ultra-relativistic Weibel instability in the fluid model for the background response. Shown is $\sigma=\operatorname{Im}(\omega) / \tilde{\omega}_{\mathrm{bg}}$, as a function of the dimensionless wavenumber, $\kappa=$ $K c / \tilde{\omega}_{\text {bg }}$, for an instability driven by two beams of equal strength (the symmetric case). Growth rates are shown for two different values of $\eta$, $\eta=0.01$ and $\eta=1$ and for a range of values of the "Mach number" $\mathcal{M}$. The different curves are labeled by the value of $\mathcal{M}$.

- Around $\kappa \sim 1$ the growth rate saturates. If $\kappa_{\max } \gg 1$ the growth rate has a nearly constant value for $1 \ll \kappa \ll \kappa_{\max }$ which, in the approximation (46), equals:

$$
\sigma \approx \frac{\kappa_{\max }}{\mathcal{M}} \approx \sqrt{\eta}
$$

- The growth rate rapidly decreases to zero at $\kappa \approx \kappa_{\max } \approx$ $\sqrt{\eta} \mathcal{M}$.

Comparing the ultra-relativistic result (46) to the classic nonrelativistic result for a cold plasma (25) we can identify the following differences:

- The cold plasma result does not have a cut-off wave number $\kappa_{\max }$.

- The denominator of the non-relativistic expression contains an extra $\tilde{\omega}_{\mathrm{pb}}^{2}$ term because one can not apply the limit $\eta \leq 1$, $\gamma_{\mathrm{b}} \gg 1$ in that case. The corresponding term in the ultrarelativistic case is $\tilde{\omega}_{\mathrm{pb}}^{2} / \gamma_{\mathrm{b}}^{2}$, which has been neglected with respect to $\tilde{\omega}_{\mathrm{bg}}^{2}$. That is allowed whenever $\eta \ll \gamma_{\mathrm{b}}^{2}$.

- In the relativistic case it is important to use the appropriate relativistic expressions for the plasma frequencies.

Note, however, that the peak value of the growth rate $\sigma \approx \sqrt{\eta}$ for the ultra-relativistic case is the relativistic equivalent for $V_{\mathrm{b}} \simeq c$ of the maximum growth rate for the non-relativistic case.

\section{Ultra-relativistic background gas}

Next we consider the case where the background plasma is described as an isotropic, ultra-relativistic gas. In almost all relevant cases one only needs to consider the electron background response (see below).

We approximate the distribution function of the background electrons by the ultra-relativistic limit (thermal energy $k_{\mathrm{b}} T \gg$ $m_{\mathrm{e}} c^{2}$ ) of the well-known Juttner distribution with temperature $T$ :

$f_{0}(\boldsymbol{p})=\frac{1}{8 \pi}\left(\frac{c}{k_{\mathrm{b}} T}\right)^{3} \exp \left(-c|\boldsymbol{p}| / k_{\mathrm{b}} T\right)$.
Substituting this into Eq. (C.7) of the Appendix (with $\left(f_{0 \mathrm{~b}}(\boldsymbol{p}) \rightarrow\right.$ $\left.f_{0}(\boldsymbol{p})\right)$ one finds (cf. Alexandrov et al. 1984; Melrose 2001)

$\alpha_{33}^{\mathrm{bg}}=\frac{\tilde{\omega}_{\mathrm{bg}}^{2} \omega}{4 K c}\left[\frac{2 \omega}{K c}+\left(1-\frac{\omega^{2}}{K^{2} c^{2}}\right) \ln \left(\frac{\omega+K c}{\omega-K c}\right)\right]$.

Here the background plasma frequency is defined as

$\tilde{\omega}_{\mathrm{bg}}^{2} \equiv \frac{4 \pi e^{2} n c^{2}}{k_{\mathrm{b}} T}$

The contribution of the ions is neglected, assuming that the ions have not thermalized yet in the shock transition.

If the frequency is purely imaginary, $\omega=\mathrm{i} \tilde{\sigma}$ as is the case here for the unstable mode, one can write $\alpha_{33}^{\text {bg }}$ as:

$$
\begin{aligned}
\alpha_{33}^{\mathrm{bg}}= & \frac{\tilde{\omega}_{\mathrm{bg}}^{2}}{2}\left[\frac{\pi}{2}\left(\frac{\tilde{\sigma}}{K c}\right)\left(1+\frac{\tilde{\sigma}^{2}}{K^{2} c^{2}}\right)-\left(\frac{\tilde{\sigma}}{K c}\right)^{2}\right. \\
& \left.-\left(\frac{\tilde{\sigma}}{K c}\right)\left(1+\frac{\tilde{\sigma}^{2}}{K^{2} c^{2}}\right) \tan ^{-1}\left(\frac{\tilde{\sigma}}{K c}\right)\right] .
\end{aligned}
$$

This follows from the representation $\tan ^{-1}(x)=(i / 2) \ln [(i+$ $x) /(i-x)]$ together with $\tan ^{-1}(x)=\pi / 2-\tan ^{-1}(1 / x)$, cf. Abramowitz \& Stegun (1970). The dispersion relation for the Weibel instability due to symmetric counterstreaming beams, $\mathcal{D}_{33}=0$, now reads:

$$
\begin{aligned}
\sigma^{2}+ & \kappa^{2}+\eta \mathcal{G}_{\mathrm{b}}+\frac{\sigma}{2 \kappa}\left[\frac{\pi}{2}\left(1+\frac{\sigma^{2}}{\kappa^{2}}\right)-\frac{\sigma}{\kappa}\right. \\
& \left.-\left(1+\frac{\sigma^{2}}{\kappa^{2}}\right) \tan ^{-1}\left(\frac{\sigma}{\kappa}\right)\right]-\frac{\eta \kappa^{2} V_{z 0}^{2}}{\sigma^{2}+\kappa^{2} V_{x 0}^{2}}=0 .
\end{aligned}
$$

Here we employ the dimensionless quantities introduced in the previous section. In the limit $\mathcal{M}^{2}=V_{z 0}^{2} / V_{x 0}^{2} \rightarrow \infty$ (cold beams) and $\sigma^{2} \ll \kappa^{2}$, this corresponds to the dispersion relation considered recently by Lyubarsky \& Eichler (2006).

The unstable modes extend from $0<\kappa<\kappa_{\max }$, where the value of $\kappa_{\max }$ follows from the condition $\sigma\left(\kappa_{\max }\right)=0$. It is easily seen that $\kappa_{\max }$ equals

$\kappa_{\max }=\sqrt{\eta\left(\mathcal{M}^{2}-\mathcal{G}_{\mathrm{b}}\right)}$

essentially the same value as obtained in the waterbag beam/fluid background model for $\eta \mathcal{M}^{2} \gg 1$, the ultra-relativistic limit.

Figure 2 shows the unstable Weibel-branch solutions of dispersion relation (53). When one compares the dispersion curves in this figure with the corresponding fluid results of Fig. 1, one finds that the curves are very similar. The physical reason is that - in both the fluid and the kinetic model - the presence of the hot background leads to a screening current, which slows the instability at small $\kappa$. In the fluid model, which can never fully capture the effect of the large velocity dispersion of the background electrons, the classical screening current leads to a diminished growth rate at wavelengths larger than the skin depth $\lambda_{\mathrm{sk}}=c / \tilde{\omega}_{\mathrm{bg}}$. In the kinetic model of this section, which takes proper account of the velocity dispersion, it is the anomalous screening current due to the velocity dispersion of the particles along $\boldsymbol{k}$ that leads to a similar effect.

We will consider the low frequency limit, $|\tilde{\sigma}| \ll K c$. In that case one has

$\alpha_{33}^{\mathrm{bg}} \simeq \frac{\pi \tilde{\omega}_{\mathrm{bg}}^{2} \tilde{\sigma}}{4 K c}=\frac{\pi \tilde{\omega}_{\mathrm{bg}}^{2}}{4}\left(\frac{\sigma}{\kappa}\right)$. 


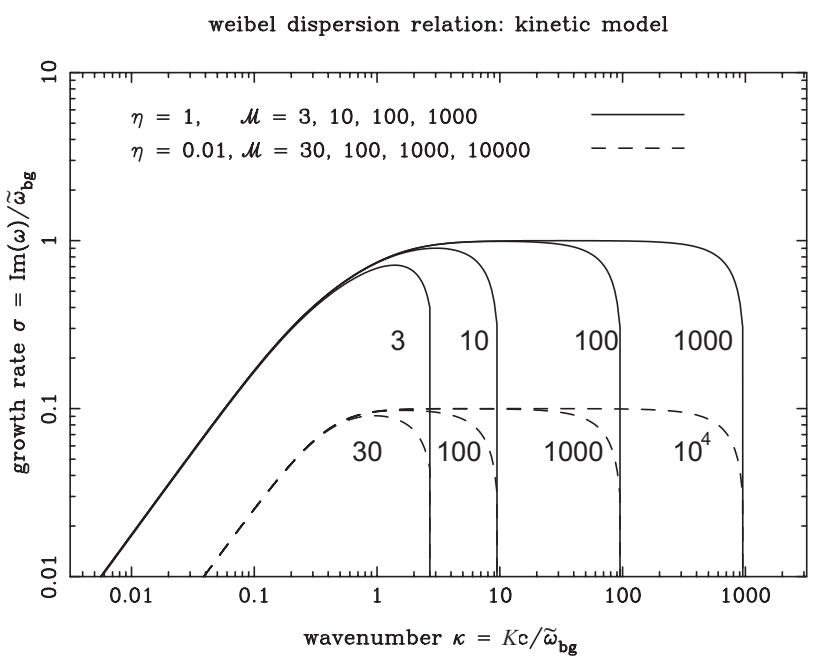

Fig. 2. The dimensionless growth rate of the ultra-relativistic Weibel instability that follows from dispersion relation (53), which employs a kinetic model for the background response. Shown is $\sigma=\tilde{\sigma} / \tilde{\omega}_{\text {bg }}$ as a function of the dimensionless wavenumber, $\kappa=K c / \tilde{\omega}_{\mathrm{bg}}$, for an instability driven by two beams of equal strength (the symmetric case). Growth rates are shown for two different values of $\eta$ and for a range of values of the "Mach number" $\mathcal{M}$. The different curves are labeled by the value of $\mathcal{M}$. The reduction in growth rate for $\kappa \ll 1$ corresponds to the effect of anomalous screening by the hot background plasma.

This result corresponds to the anomalous skin effect in an isotropic ultra-relativistic thermal plasma. Taking the ultrarelativistic limit for the beams, in effect putting $\mathcal{G}_{\mathrm{b}} \approx 0, V_{z 0} \approx$ $1 \gg V_{x 0}$ and $\eta \mathcal{M}^{2} \gg 1$, one can replace the full dispersion relation (53) by the simpler relation

$\kappa^{2}\left(\sigma^{2}+\frac{\kappa^{2}}{\mathcal{M}^{2}}-\eta\right)+\frac{\pi}{4} \frac{\sigma}{\kappa}\left(\sigma^{2}+\frac{\kappa^{2}}{\mathcal{M}^{2}}\right)=0$.

It is possible to write down a rather complicated expression for the analytical solution of the cubic Eq. (56) for the dimensionless growth rate $\sigma$. However, the close similarity (see Fig. 2) with the results for the growth rate obtained with a fluid treatment of the background plasma suggests that the following interpolation formula applies, asymptotically exact for both $\kappa^{2} \ll 1$ and $\kappa^{2} \gg$ 1 when $\eta \leq 1$ :

$\sigma^{2} \simeq \frac{\kappa^{2}\left(\kappa_{\max }^{2}-\kappa^{2}\right)}{\mathcal{M}^{2}\left(\kappa_{\mathrm{s}}^{2}+\kappa^{2}\right)}$.

The screening wavenumber $\kappa_{\mathrm{s}}$, which in this case quantifies the anomalous skin effect, follows from a consideration of the solution of dispersion relation (56) in the limit $\kappa^{2} \ll 1, \sigma^{2} \ll 1$ and $\kappa_{\max }^{2} \approx \eta \mathcal{M}^{2} \gg 1$ :

$\sigma^{2} \approx\left(\frac{4 \eta}{\pi}\right)^{2 / 3} \kappa^{2}$

Comparing this with (57) in this limit,

$\sigma^{2} \approx \frac{\kappa^{2} \kappa_{\max }^{2}}{\kappa_{\mathrm{S}}^{2} \mathcal{M}^{2}}=\frac{\eta \kappa^{2}}{\kappa_{\mathrm{s}}^{2}}$

one finds that one must have

$\kappa_{\mathrm{S}}=\left(\frac{\pi}{4}\right)^{1 / 3} \eta^{1 / 6}$.
This defines the dimensionless screening wave number $\kappa_{\mathrm{S}}$ for the kinetic model for the background plasma. The fluid approximation for the background corresponds to putting $\kappa_{\mathrm{s}}=1$. Anomalous screening is important when $\kappa \leq \kappa_{\mathrm{s}}$. Note that the value of $\kappa_{\mathrm{S}}$ depends only weakly on the strength of the beams, and will be close to unity unless $\eta$ is very small or very large. Figure 3 compares the growth rate as calculated from the various dispersion relations for the Weibel instability, both exact and approximate. The comparison is shown for two cases with an identical value of $\kappa_{\max } \approx 33$ : for a relatively dense beam $(\eta=1)$, and for an underdense beam with $\eta=0.01$. For $\kappa \gg 1$ there is little difference as the screening currents of the hot background are relatively unimportant. In the case $\eta=1$ the largest differences occur between the various approaches, but the difference between the growth rate as calculated from the fluid model and the kinetic model for the background is no more than a factor 2 . In the case $\eta=0.01$ the difference between the two models is somewhat more pronounced as the screening wavenumber squared, $\kappa_{\mathrm{s}}^{2} \approx 0.18$, becomes significantly smaller than unity. The growth rates as obtained numerically from the exact dispersion relations, and the approximate growth rates obtained from the approximate relations (46) and (57), agree fairly well for $\eta=1$, and give an excellent approximation in the case $\eta=0.01$. This is to be expected in view of the approximation $\sigma^{2} \ll \kappa^{2}$ employed in the derivation of the approximate growth rates, as the maximum growth rate in all cases is $\sigma_{\max } \sim \sqrt{\eta}$.

\section{Magnetized Weibel instability}

The previous calculations have been for an unmagnetized plasma. In this section we briefly consider the magnetized case, in order to compare the results obtained using our formalism with those of Tautz \& Schlickeiser (2006) and to show that, for typical parameters, the unmagnetized case applies to the Weibel instability near ultra-relativistic shocks propagating into the interstellar (or circumstellar) medium.

We can only consider the case where the Lorentz force on all species in the unperturbed plasma vanishes: $F^{\mu v} U_{\mathrm{sv}}=0$. This implies that there is no ambient electric field in the laboratory frame, and that we are dealing with two equal counterstreaming beams, $\Delta=0$, with the beam velocity aligned with the magnetic field. These conditions are rather restrictive. A calculation of the response of the plasma in the fluid approximation (see Appendix D) leads to an instability of the ordinary electromagnetic mode in a plasma, cf. Tautz \& Schlickeiser (2006). In the ordinary mode the wave electric field is along the beam direction/ambient magnetic field in the lab frame so that $\tilde{\boldsymbol{E}}=\tilde{E} \hat{z}$. As before, we choose a wave vector in the laboratory frame perpendicular to the beam direction: $\boldsymbol{k}=K \hat{\boldsymbol{x}}$. The presence of the magnetic field modifies dispersion relation (27) to (see Eq. (D.6) of Appendix D):

$\omega^{2}=K^{2}+\tilde{\omega}_{\mathrm{bg}}^{2}+\tilde{\omega}_{\mathrm{pb}}^{2}\left(1-\frac{\left(\omega^{2}-K^{2}\right) \tilde{V}_{\mathrm{b}}^{2}}{\omega^{2}-K^{2} \tilde{C}_{\mathrm{b}}^{2}-\tilde{\Omega}_{\mathrm{b}}^{2}}\right)$.

Here

$\tilde{\Omega}_{\mathrm{b}}=\frac{q_{\mathrm{b}} B}{\gamma_{\mathrm{b}} m_{\mathrm{b}} h_{\mathrm{b}} \sqrt{1-V_{\mathrm{b}}^{2} C_{\mathrm{b}}^{2}}} \equiv \frac{\Omega_{\mathrm{b}}}{\gamma_{\mathrm{b}} \sqrt{1-V_{\mathrm{b}}^{2} C_{\mathrm{b}}^{2}}}$,

is an effective gyration frequency of the beam plasma, with $B$ the magnetic field strength and $\Omega_{\mathrm{b}} \equiv q_{\mathrm{b}} B / m_{\mathrm{b}} h_{\mathrm{b}}$. For field-aligned beams the magnetic field is the same in the lab frame and in the two respective rest frames of the forward and backward beam. In the limit of a vanishing beam temperature $\left(C_{b}=0\right.$, 

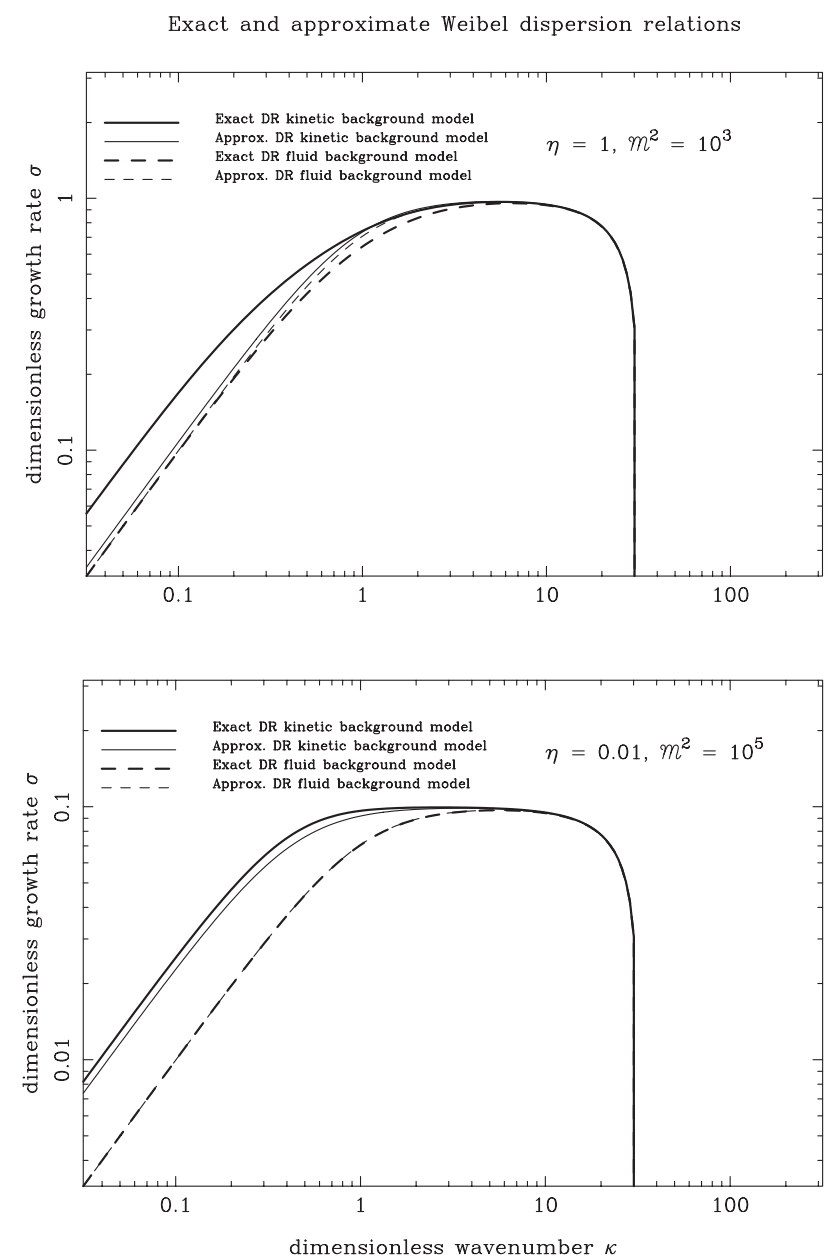

Fig. 3. A comparison of the growth rates calculated from the fluid and the kinetic model for the background response, and from the approximate dispersion relations in both these models: Eqs. (46) and (57). Dispersion curves are shown in two cases: $\eta=1$ (top figure) and $\eta=0.01$ (bottom figure), with $\mathcal{M}^{2}=10^{3}$ and $\mathcal{M}^{2}=10^{5}$ respectively, so that the value of the maximum unstable wavenumber, $\kappa_{\max } \approx \sqrt{\eta} \mathcal{M}$, is identical in both cases.

$\left.h_{\mathrm{b}}=1\right)$, of low frequency $\left(\left|\omega^{2}\right| \ll K^{2}\right)$ perturbations and for a non-relativistic beam velocity (so that $V_{\mathrm{b}} \ll 1, \gamma_{\mathrm{b}} \simeq 1$ and $\tilde{\Omega}_{\mathrm{b}} \simeq q_{\mathrm{b}} B / m_{\mathrm{b}}$ ) dispersion relation (61) reduces to the dispersion relation derived by Tautz \& Schlickeiser (2006), their Eq. (19), in the corresponding case.

Dispersion relation (61) for the magnetized Weibel instability can again be written in the form of Eq. (34): $\omega^{4}-\mathcal{B}(K) \omega^{2}+$ $C(K)=0$. The relevant coefficients $\mathcal{B}(K)$ and $C(K)$ are listed in Eqs. (D.8) and (D.9) of Appendix D.

The most important effect of the magnetic field is a modification of the range of unstable wavenumbers that follows from the instability condition $C(K)<0$. It introduces a wavenumber $K_{-}$, below which the magnetic field stabilizes the Weibel instability. Unstable modes occur for $K_{-}<|K|<K_{+}$, where $K_{-}\left(K_{+}\right)$is the smaller (larger) real root of $C(K)=0$. From expression (D.9) for $C(K)$ one finds that the two limiting wavenumbers $K_{ \pm}$are given by:

$$
\begin{aligned}
K_{ \pm}^{2}= & \frac{K_{\max }^{2}-K_{\mathrm{B}}^{2}}{2} \\
& \pm \sqrt{\left(\frac{K_{\mathrm{max}}^{2}-K_{\mathrm{B}}^{2}}{2}\right)^{2}-\left(\tilde{\omega}_{\mathrm{pb}}^{2}+\tilde{\omega}_{\mathrm{bg}}^{2}\right) K_{\mathrm{B}}^{2}} .
\end{aligned}
$$

The wavenumber $K_{\mathrm{B}}$ is defined by

$K_{\mathrm{B}} \equiv \frac{\left|\tilde{\Omega}_{\mathrm{b}}\right|}{\tilde{C}_{\mathrm{b}}}=\frac{\left|q_{\mathrm{b}}\right| B}{m_{\mathrm{b}} h_{\mathrm{b}} C_{\mathrm{b}}}$,

and $K_{\max }$ is the maximum unstable wavenumber in the field-free case as defined in Eq. (38). The Weibel instability disappears in the magnetized case when the unstable wavenumber range shrinks to zero. This happens when the argument of the square root in expression (63) vanishes or becomes negative for

$K_{\mathrm{B}}>\sqrt{K_{\mathrm{max}}^{2}+4\left(\tilde{\omega}_{\mathrm{pb}}^{2}+\tilde{\omega}_{\mathrm{bg}}^{2}\right)}-2 \sqrt{\tilde{\omega}_{\mathrm{pb}}^{2}+\tilde{\omega}_{\mathrm{bg}}^{2}}$.

The presence of a magnetic field also raises the threshold velocity: there is only an instability at a given wavenumber $K$ if

$\left(\frac{V_{\mathrm{b}}}{C_{\mathrm{b}}}\right)^{2}=\mathcal{M}^{2}>\frac{\left(K^{2}+K_{\mathrm{B}}^{2}\right)\left(K^{2}+\tilde{\omega}_{\mathrm{pb}}^{2}+\tilde{\omega}_{\mathrm{bg}}^{2}\right)}{K^{2} \tilde{\omega}_{\mathrm{pb}}^{2}}$.

This last condition is analogous to condition (20b) of Tautz \& Schlickeiser (2006) (after correcting a misprint), who only consider the non-relativistic case.

For the remainder of this section we consider the ultrarelativistic limit with $\mathcal{M}^{2} \gg 1, V_{\mathrm{b}} \simeq 1 \gg C_{\mathrm{b}} \simeq 1 / \mathcal{M}$. Defining the dimensionless limiting wavenumbers $\kappa_{ \pm}=K_{ \pm} / \tilde{\omega}_{\text {bg }}$ and a dimensionless gyration frequency $\sigma_{\mathrm{B}}=\tilde{\Omega}_{\mathrm{b}} / \tilde{\omega}_{\mathrm{bg}}$, the growth rate of the unstable solution can be represented by the analogue for the magnetized case of relation (45):

$$
\begin{aligned}
\sigma^{2}= & \sqrt{\left(\frac{1+\kappa^{2}+\sigma_{\mathrm{B}}^{2}}{2}\right)^{2}+\frac{\left(\kappa^{2}-\kappa_{-}^{2}\right)\left(\kappa_{+}^{2}-\kappa^{2}\right)}{\mathcal{M}^{2}}} \\
& -\frac{1+\kappa^{2}+\sigma_{\mathrm{B}}^{2}}{2} .
\end{aligned}
$$

The magnetized equivalent of the approximate dispersion relation (46), valid when $\left(1+\kappa^{2}+\sigma_{\mathrm{B}}^{2}\right)^{2} \gg 4\left(\kappa^{2}-\kappa_{-}^{2}\right)\left(\kappa_{+}^{2}-\kappa^{2}\right) / \mathcal{M}^{2}$, reads:

$\sigma^{2} \approx \frac{\left(\kappa^{2}-\kappa_{-}^{2}\right)\left(\kappa_{+}^{2}-\kappa^{2}\right)}{\mathcal{M}^{2}\left(1+\kappa^{2}+\sigma_{\mathrm{B}}^{2}\right)}$

In the ultra-relativistic limit, with $C_{\mathrm{b}} \ll 1$ and $\mathcal{M}^{2} \gg 1$, one has $K_{\mathrm{B}} \simeq \mathcal{M}\left(\Omega_{\mathrm{b}} / \gamma_{\mathrm{B}}\right)$ and $K_{\mathrm{max}} \simeq \mathcal{M} \tilde{\omega}_{\mathrm{pb}}$. If $K_{\mathrm{max}}^{2} \gg \tilde{\omega}_{\mathrm{pb}}^{2}+\tilde{\omega}_{\mathrm{bg}}^{2}$ (or equivalently: $\eta \mathcal{M}^{2} \gg 1+\eta$ ) the Weibel instability vanishes according to $(65)$ if the magnetic field gets so large that $K_{\mathrm{B}} \approx$ $K_{\max }$, or equivalently

$\frac{\left|\Omega_{\mathrm{b}}\right|}{\gamma_{\mathrm{b}}} \simeq \frac{\left|q_{\mathrm{b}}\right| B}{\gamma_{\mathrm{b}} m_{\mathrm{b}}} \approx \tilde{\omega}_{\mathrm{pb}}$.

In Fig. 4 we show the solution for the dimensionless growth rate $\sigma(\kappa)$ of the unstable Weibel mode (ordinary mode) in the magnetized case.

The behavior of the growth rate is analogous to the limiting expressions derived recently by Stockem et al. (2006) for the filamentation instability due to field-aligned beams in a magnetized and cold plasma $\left(C_{\mathrm{b}}=0\right)$. These authors use an expansion of the full dispersion relations near the points $\omega^{2}=0$ that separate the stable $\left(\omega^{2}>0\right)$ and unstable $\left(\omega^{2}<0\right)$ wavelength regions, see Tautz et al. (2007) for details. They consider multi-species (ions and electrons) beams without a thermal background $\left(\tilde{\omega}_{\mathrm{bg}}=0\right)$ in the the non-relativistic limit $V_{\mathrm{b}} \ll c$. For 


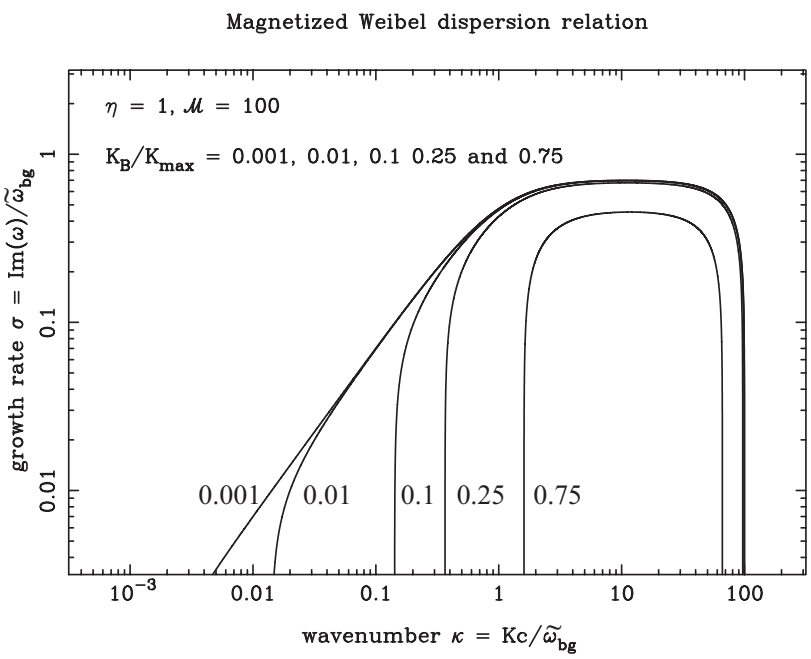

Fig. 4. The dimensionless growth rate $\sigma$ for the magnetized Weibel instability for field-aligned beams as a function of dimensionless wavenumber $\kappa$ as calculated from the fluid model, for $\eta=1, \mathcal{M}=100$, and $K_{\mathrm{B}} / K_{\max }=0.001,0.01,0.1,0.25$, and 0.75 , corresponding to an increasing magnetic field. The different curves are labeled by the value of $K_{\mathrm{B}} / K_{\max }$. The modification Weibel with respect to the field-free case becomes important for $K_{\mathrm{B}} \sim K_{\max }$.

$V_{\mathrm{b}} \ll c$ the approximation $\omega^{2}=-\tilde{\sigma}^{2} \simeq C(K) / \mathcal{B}(K)$ is universally valid. Using the expressions (D.8) and (D.9) of Appendix D with $\tilde{C}_{\mathrm{b}}=0$ and $\tilde{V}_{\mathrm{b}} \simeq V_{\mathrm{b}} \ll 1$ one finds, using dimensional units, reinstating $c$ for clarity and retaining the background term:

$\tilde{\sigma}^{2}=\tilde{\omega}_{\mathrm{pb}}^{2}\left\{\frac{\left(V_{\mathrm{b}}^{2}-V_{\mathrm{Ab}}^{2}\right)\left(K^{2}-K_{-}^{2}\right)}{\tilde{\omega}_{\mathrm{bg}}^{2}+\tilde{\omega}_{\mathrm{pb}}^{2}+\Omega_{\mathrm{b}}^{2}+K^{2} c^{2}}\right\}$.

Here $V_{\mathrm{Ab}}=B / \sqrt{4 \pi n_{\mathrm{b}} m_{\mathrm{b}}}=c \Omega_{\mathrm{b}} / \tilde{\omega}_{\mathrm{pb}}$ is the Alfvén velocity associated with the beam particles, and $\tilde{\omega}_{\mathrm{pb}}=\sqrt{4 \pi q_{\mathrm{b}}^{2} n_{\mathrm{b}} / m_{\mathrm{b}}}$, $\Omega_{\mathrm{b}}=q_{\mathrm{b}} B / m_{\mathrm{b}} c$ in this limit. Expression (63) for $K_{-}$is replaced by $K_{-}=\left(\Omega_{\mathrm{b}} \sqrt{\tilde{\omega}_{\mathrm{pb}}^{2}+\tilde{\omega}_{\mathrm{bg}}^{2}}\right) /\left(\tilde{\omega}_{\mathrm{pb}} \sqrt{V_{\mathrm{b}}^{2}-V_{\mathrm{Ab}}^{2}}\right)$. The expression for the growth rate scales exactly the same manner as the expressions in Stockem et al. (2006), their Eqs. (31) and (33), in the appropriate limits: $\sigma \propto \sqrt{K^{2}-K_{-}^{2}}$ near instability threshold and $\sigma \sim$ constant for large $K$. The threshold condition is also the same as found by these authors: there can only be an instability when $V_{\mathrm{b}}>V_{\mathrm{Ab}}$.

\subsection{When can non-magnetic results be used?}

We are mostly interested in the application of the Weibel instability to the ultra-relativistic shocks associated with gamma ray bursts. The influence of the magnetic field on the Weibel instability will be small if $K_{-} \ll \tilde{\omega}_{\text {bg }}\left(\kappa_{-} \ll 1\right)$, so that both the maximum growth rate and the range of unstable wavenumbers are comparable with their values in the field-free case, see Fig. 4. From the discussion above, and in particular from Eq. (63), it is clear that this situation occurs if $K_{\mathrm{B}} \ll K_{\max }$. In the ultrarelativistic regime, the growth rate of the instability, $\tilde{\sigma}=\operatorname{Im}(\omega)$, is then roughly constant over a wide wavelength range, with

$\tilde{\sigma} \approx \tilde{\omega}_{\mathrm{pb}} V_{\mathrm{b}}$ for $\tilde{\omega}_{\mathrm{bg}}<K<\tilde{\omega}_{\mathrm{pb}} \mathcal{M}$, assuming $\eta \mathcal{M}^{2} \gg 1$. This should be compared with the gyration frequency of beam particles, which for a relativistic cold beam with $C_{\mathrm{b}} \ll 1$ is

$\left|q_{\mathrm{b}}\right| B / \gamma_{\mathrm{b}} m_{\mathrm{b}} \approx\left|\Omega_{\mathrm{b}}\right| / \gamma_{\mathrm{b}}$,

The ratio of the gyration frequency and typical Weibel growth rate is therefore

$\frac{\left|\Omega_{\mathrm{b}}\right|}{\gamma_{\mathrm{b}} \tilde{\sigma}} \approx \frac{\left|\Omega_{\mathrm{b}}\right|}{\gamma_{\mathrm{b}} \tilde{\omega}_{\mathrm{pb}} V_{\mathrm{b}}} \approx \frac{K_{\mathrm{B}}}{K_{\max }}$.

This shows that the condition $K_{\mathrm{B}} \ll K_{\max }$ also ensures that the growth rate of the instability is much larger than the gyration frequency of the beam particles.

Taking the beam material to be the unshocked (cold) hydrogen plasma interpenetrating the (hot) shocked plasma, the ratio of the beam gyration frequency and Weibel instability growth rate equals:

$\frac{\left|\Omega_{\mathrm{b}}\right|}{\gamma_{\mathrm{b}} \tilde{\omega}_{\mathrm{pb}}} \approx\left\{\begin{array}{l}3 \times 10^{-4} \frac{B_{\mu \mathrm{G}}}{\gamma_{\mathrm{b}} \sqrt{n_{\mathrm{e}}}} \text { (electrons) } \\ 7 \times 10^{-5} \frac{B_{\mu \mathrm{G}}}{\gamma_{\mathrm{b}} \sqrt{n_{\mathrm{p}}}} \text { (protons) }\end{array}\right.$

Here $B_{\mu \mathrm{G}}$ is the strength of the pre-existing magnetic field in the shock transition layer in units of micro-Gauss, and $n_{\mathrm{e}}\left(n_{\mathrm{p}}\right)$ is the proper density of the electron (proton) beam. This proper density is always comparable to the density of the particles in the preshock medium. The magnetic field strength in the transition layer depends on the orientation of the pre-shock magnetic field. For a magnetic field parallel to the shock normal (the case for which the magnetized Weibel dispersion relation was derived) there is no field amplification due to shock compression, and the ambient magnetic field in the transition layer equals the upstream field, even after a Lorentz transformation to the rest frame of the hot post-shock material. In that case, the ratio $\left|\Omega_{\mathrm{b}}\right| / \gamma_{\mathrm{b}} \tilde{\omega}_{\mathrm{pb}}$ is always much smaller than unity for typical parameters: $B_{\mu \mathrm{G}} \sim 1-100$, $\gamma_{\mathrm{b}} \sim \gamma_{\mathrm{sh}} / \sqrt{2} \sim 100$ and $n_{\mathrm{e}, \mathrm{p}} \sim 1-100 \mathrm{~cm}^{-3}$. That implies that in this case the effect of the magnetic field can be neglected altogether: the growth of the Weibel Instability is so rapid that particles do not get a chance to start gyrating around the ambient (pre-existing) magnetic field. Their dynamics can be described as being unmagnetized, and the range of unstable wavenumbers and instability growth rate are hardly affected in this case.

If there is a component $B_{\mathrm{t}}$ of the magnetic field along the shock surface, it could be amplified considerably, with $B_{\mathrm{t} 2} \approx$ $2 \sqrt{2} \gamma_{\mathrm{sh}} B_{\mathrm{t} 1}$ upon completion of the shock transition according to the relativistic MHD shock jump conditions (see Sect. 1). But even then one has $B_{\mathrm{t} 2} / \gamma_{\mathrm{b}} \approx \sqrt{2} B_{\mathrm{t} 2} / \gamma_{\mathrm{sh}} \sim 4 B_{\mathrm{t} 1}$. Here we have used that $\gamma_{\mathrm{b}} \approx \gamma_{\mathrm{rel}} \approx \gamma_{\mathrm{sh}} / \sqrt{2}$, where $\gamma_{\text {rel }}$ is the Lorentz factor associated with the relative motion between the up- and downstream flow. We reiterate however that this case, where the beams are not (anti)parallel with the magnetic field, has not been treated here. In fact, the plasma dynamics near a perpendicular relativistic shock may very well involve quite different plasma instabilities, in particular for the thermalization of the heavier ions, such as the ion-driven gyroresonant instability discussed by Spitkovsky \& Arons (2005) in the context of the relativistic shocks in the pulsar wind that creates the Crab Nebula.

Some support for the relative unimportance of the magnetic field in the relativistic case with $K_{\mathrm{B}} \ll K_{\max }$ comes from the simulations of Hededal \& Nishikawa (2005). Because of numerical limitations they can only follow the electron-driven Weibel 
instability. In the case of field-aligned beams they find that the Weibel instability develops unhindered if $\tilde{\omega}_{\mathrm{pb}} \gg \Omega_{\mathrm{b}} / \gamma_{\mathrm{b}}$, and that the instability is totally supressed if the beam plasma frequency and the beam gyrofrequency become of equal: $\tilde{\omega}_{\mathrm{pb}} \sim \Omega_{\mathrm{b}} / \gamma_{\mathrm{b}}$ in our notation. In the case of a magnetic field that is perpendicular to the beam they find a significant modification of the plasma behavior when $\Omega_{\mathrm{b}} / \gamma_{\mathrm{b}}>0.05 \tilde{\omega}_{\mathrm{pb}}$.

It should be pointed out that a similar conclusion about the limited importance of an ambient magnetic field does not necessarily hold for the non-relativistic version of the Weibel instability. If the beam velocity satisfies $V_{\mathrm{b}} \ll c$, the maximum growth rate (for a cold beam) is (see Eq. (25)):

$\tilde{\sigma}=\tilde{\omega}_{\mathrm{pb}}\left(\frac{V_{\mathrm{b}}}{c}\right)$.

The ratio of this growth rate and the typical gyration frequency $\Omega_{\mathrm{b}}=q_{\mathrm{b}} B / m_{\mathrm{b}} c$ of the beam particles is now

$\frac{\tilde{\sigma}}{\Omega_{\mathrm{b}}} \approx \frac{V_{\mathrm{b}}}{V_{\mathrm{Ab}}}$,

where $V_{\mathrm{Ab}} \equiv B / \sqrt{4 \pi n_{\mathrm{b}} m_{\mathrm{b}}}$ is an Alfvén velocity associated with the beams. In this case one needs $V_{\mathrm{b}} \gg V_{\mathrm{Ab}}$ in order for the unmagnetized result to apply. This is not guaranteed, especially for light particles such as electrons. Also, the long-wavelength cutoff of the unstable wavelength range at $K=K_{-}$can be important in this case.

\section{The asymmetric case}

With the exception of our discussion of the cold beam/cold background case in Sect. 4, we have so far treated the symmetric case of the Weibel instability driven by two identical but counterstreaming beams. In that case the beams carry no net current and the Weibel instability is purely transverse in the rest frame of the background plasma. In this section we briefly consider the asymmetric case with $\Delta \neq 0$, using the waterbag approximation for the beams.

We neglect the drift in the background plasma which occurs in the asymmetric case if the beams carry only one sign of charge. This drift supplies the return current to the beam current which is needed in the steady state. The effect of the drift will be small if the beam density is small compared to the density of the background plasma. If the beams themselves are electrically neutral, containing an equal amount of positively and negatively charged particles, our results apply after a straightforward generalization to a multi-species beam.

We will first transform dispersion relation (20) into a more convenient form. The longitudinal dielectric response of both background plasma and beams is contained in $\mathcal{D}_{11}(\omega, K)$, which is (see Eq. (C.12) of Appendix C)

$\mathcal{D}_{11}(\omega, K)=-|k \cdot k|\left(1-\sum_{s \in \mathrm{bg}} \frac{\tilde{\omega}_{\mathrm{ps}}^{2}}{\omega^{2}-K^{2} C_{\mathrm{s}}^{2}}-\frac{\hat{\omega}_{\mathrm{pb}}^{2}}{\omega^{2}-K^{2} V_{x 0}^{2}}\right)$.

The off-diagonal term $\mathcal{D}_{31}$ gives the coupling between the electrostatic and transverse fields. We now assume that the background plasma is relativistically hot, with $C_{\mathrm{s}} \approx 1 / \sqrt{3}$ for all species, and that the beam is ultra-relativistic so that $V_{x 0} \ll$ $V_{z 0} \approx 1$. In the low-frequency limit $(\omega \ll K)$ one has

$\mathcal{D}_{11}(\omega, K) \approx-K^{2}\left(1+\frac{3 \tilde{\omega}_{\mathrm{bg}}^{2}}{K^{2}}-\frac{\hat{\omega}_{\mathrm{pb}}^{2}}{\omega^{2}-K^{2} V_{x 0}^{2}}\right)$,
$\mathcal{D}_{33}(\omega, K) \approx K^{2}+\tilde{\omega}_{\mathrm{bg}}^{2}+\frac{\hat{\omega}_{\mathrm{pb}}^{2} K^{2}}{\omega^{2}-K^{2} V_{x 0}^{2}}$,

$\mathcal{D}_{31}(\omega, K) \approx \Delta\left(\frac{\hat{\omega}_{\mathrm{pb}}^{2} K^{2}}{\omega^{2}-K^{2} V_{x 0}^{2}}\right)$,

where we have put $V_{z 0}=1$ everywhere.

We define a new dimensionless variable to parameterize the solution of the dispersion relation (20),

$\mathcal{Z}(\omega, K) \equiv \frac{\omega^{2}-K^{2} V_{x 0}^{2}}{\hat{\omega}_{\mathrm{pb}}^{2}}$,

and introduce the two quantities

$\mathcal{Z}_{1}(K)=\left(1+\frac{3 \tilde{\omega}_{\mathrm{bg}}^{2}}{K^{2}}\right)^{-1}=\frac{\kappa^{2}}{3+\kappa^{2}} ;$

$\mathcal{Z}_{2}(K)=\frac{K^{2}}{\tilde{\omega}_{\mathrm{bg}}^{2}+K^{2}}=\frac{\kappa^{2}}{1+\kappa^{2}}$.

The dispersion relation (20) can be written in the form

$\left(\mathcal{Z}(\omega, K)-\mathcal{Z}_{1}\right)\left(\mathcal{Z}(\omega, K)+\mathcal{Z}_{2}\right)+\Delta^{2} \mathcal{Z}_{1} \mathcal{Z}_{2}=0$

The general solution for $\mathcal{Z}(\omega, K)$ in the asymmetric case reads

$\mathcal{Z}_{ \pm}(\omega, K)=\frac{\mathcal{Z}_{1}-\mathcal{Z}_{2}}{2} \pm \sqrt{\left(\frac{\mathcal{Z}_{1}+\mathcal{Z}_{2}}{2}\right)^{2}-\Delta^{2} \mathcal{Z}_{1} \mathcal{Z}_{2}}$

which implicitly determines the wave frequency.

For the choice of parameters considered here one has $\boldsymbol{Z}_{2}>$ $\mathcal{Z}_{1}$ and therefore $\mathcal{Z}_{+}>0$ and $\mathcal{Z}_{-} \leq 0$. As the frequency of the two solutions satisfies

$\omega_{ \pm}^{2}=\hat{\omega}_{\mathrm{pb}}^{2} \mathcal{Z}_{ \pm}+K^{2} V_{x 0}^{2}$

the potentially unstable Weibel branch corresponds with the solution branch $\mathcal{Z}_{-}$, with a corresponding frequency $\omega_{-}$.

The solution corresponding with $\mathcal{Z}_{+}$is stable. It corresponds to the stable transverse mode with $\tilde{\boldsymbol{E}} \perp \boldsymbol{V}_{\mathrm{b}}$ that was mentioned in Sect. 3. The unstable Weibel branch has a dimensionless growth rate $\sigma^{2} \equiv-\omega_{-}^{2} / \tilde{\omega}_{\text {bg }}^{2}=-\eta \mathcal{Z}_{-}-\kappa^{2} V_{x 0}^{2}$, which is:

$\sigma^{2}=\frac{\eta}{2}\left\{\sqrt{\left(\mathcal{Z}_{1}+\mathcal{Z}_{2}\right)^{2}-4 \Delta^{2} \mathcal{Z}_{1} \mathcal{Z}_{2}}-\left(\mathcal{Z}_{1}-\mathcal{Z}_{2}\right)\right\}-\kappa^{2} V_{x 0}^{2}$

This solution is represented graphically in Fig. 5.

We now consider a number of specific cases. In the symmetric case $(\Delta=0)$ the transverse Weibel mode corresponds to the solution $\mathcal{Z}_{-}=-\mathcal{Z}_{2}$, with a frequency that satisfies $\omega_{-}^{2}=-\hat{\omega}_{\mathrm{pb}}^{2} \mathcal{Z}_{2}+K^{2} V_{x 0}^{2}$. This is the case treated in Sect. 7, which leads to an instability if $\mathcal{Z}_{2}>K^{2} V_{x 0}^{2} / \hat{\omega}_{\mathrm{pb}}^{2}$. In the case of a single beam $(\Delta=1)$ in a relativistically hot background one has $\mathcal{Z}_{-}=\mathcal{Z}_{1}-\mathcal{Z}_{2}$ and the dimensionless growth rate becomes

$\sigma^{2}=\kappa^{2}\left(\frac{2 \eta}{\left(1+\kappa^{2}\right)\left(3+\kappa^{2}\right)}-V_{x 0}^{2}\right)$.

This case is unstable for wavenumbers satisfying

$\kappa<\kappa_{\max }(\Delta=1) \equiv\left(\sqrt{1+2 \eta \mathcal{M}^{2}}-2\right)^{1 / 2}$.

One can find a general expression for the maximum unstable wavenumber for arbitrary $-1 \leq \Delta \leq 1$ by putting $\sigma=0$. We 


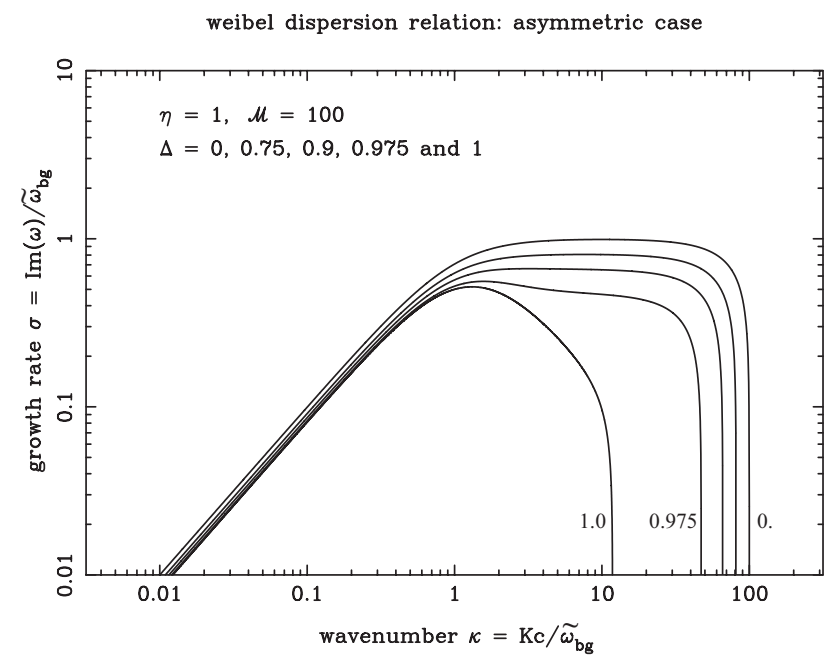

Fig. 5. The dimensionless growth rate of the Weibel instability, $\sigma=$ $\operatorname{Im}(\omega) / \tilde{\omega}_{\text {bg }}$, as a function of the dimensionless wavenumber, $\kappa=$ $K c / \tilde{\omega}_{\text {bg }}$, in the case of unequal beam densities. Growth rates are shown for $\eta=1$ and $\mathcal{M}=100$, and for a range of values of the asymmetry parameter $\Delta$ as defined in Eq. (19). The different curves are labeled by the corresponding value of $\Delta$.

give the result in the limit of a beam velocity close to light speed $\left(1-V_{z 0} \ll 1\right)$ so that $V_{x 0} \approx 1 / \mathcal{M} \ll 1$ :

$\kappa_{\max }(\Delta)=\left(\sqrt{\left(\eta \mathcal{M}^{2}+1\right)^{2}-\eta^{2} \Delta^{2} \mathcal{M}^{4}}-2\right)^{1 / 2}$.

The asymmetry has the effect of shrinking the range of unstable wavenumbers with respect to the symmetric case of $\Delta=0$. The condition $\kappa_{\max }^{2}>0$ shows that the Weibel Instability only occurs if

$\eta \mathcal{M}^{2}>\frac{\sqrt{4-3 \Delta^{2}}-1}{1-\Delta^{2}}$.

For the symmetric case $(\Delta=0)$ one finds $\eta \mathcal{M}^{2}>1$, while for the case of a single beam $(\Delta=1)$ Eq. (86) gives $\eta \mathcal{M}^{2}>3 / 2$.

If the beams are sufficiently strong, so that $\eta \mathcal{M}^{2} \gg 1$ and $\kappa_{\max }(\Delta) \gg 1$, one can approximate dispersion relation (84) in the range $\kappa^{2} \gg 1$ by using $\mathcal{Z}_{1} \approx \mathcal{Z}_{2} \approx 1$ :

$\sigma^{2} \approx \eta \sqrt{1-\Delta^{2}}-\frac{\kappa^{2}}{\mathcal{M}^{2}}$

This equation clearly shows that the main effect of the asymmetry is to suppress the maximum growth rate and maximum wave number (Fig. 5). However, the effects are small unless $\Delta$ is very close to 1 .

\section{Discussion and conclusions}

We have discussed the Weibel instability of relativistic beams in a relativistically hot background plasma. We based our calculations on the first principles of the fluid and the Vlasov description of the plasma. Our calculations generalize and extend the results in the literature by presenting a detailed analysis of the dispersion relation in tensor form. Our aims have been, in particular:

- to obtain accurate expressions to use in further non-linear calculations, which we present in the companion paper (Achterberg et al. 2006);
- to determine the basic parameters that characterize the dispersion in the plasma;

- and to estimate whether background magnetic fields or asymmetry play an important role.

We have shown that the linear growth rate in the relativistic limit is characterized by only two parameters in the symmetric case of identical but counterstreaming beams: the ratio $\eta=\tilde{\omega}_{\mathrm{pb}}^{2} / \tilde{\omega}_{\mathrm{bg}}^{2}$ of the effective plasma frequencies of beams and background plasma, and an effective Mach number $\mathcal{M}$, which is a measure for the ratio of the momentum of beam particles in the beam direction and the typical momentum of the thermal motion (velocity dispersion) in the direction along the wave vector. The latter component is a measure of the pressure in the beam plasma. These conclusions are independent of the precise model, fluid or kinetic, that is used to describe the beam-plasma system.

For ultra-relativistic beams $\left(\gamma_{\mathrm{b}} \gg 1\right)$ the maximum growth rate is $\operatorname{Im}(\omega) \approx \sqrt{\eta} \tilde{\omega}_{\text {bg }}$, and the maximum unstable wavenumber is $K_{\max } \approx \sqrt{\eta} \mathcal{M}^{2}\left(\tilde{\omega}_{\mathrm{bg}} / c\right)$.

The presence of an ambient magnetic field does not change the behavior of the Weibel instability unless the field is so strong that the beam plasma frequency and the gyration frequency of the beam particles become comparable in magnitude: $\tilde{\omega}_{\mathrm{pb}} \simeq$ $\left|q_{\mathrm{b}}\right| B / \gamma_{\mathrm{b}} m_{\mathrm{b}}$. A similar conclusion was reached by Hededal \& Nishikawa (2005) on the basis of numerical simulations. For the typical parameters associated with the ultra-relativistic shocks of gamma ray bursts (external shocks) that propagate into the interstellar or circumstellar medium the unmagnetized treatment of the Weibel instability is a good approximation.

Tautz \& Lerche (2006) have recently discussed the influence of the ambient magnetic field on the stability properties of relativistic beam plasmas with a general distribution function $f_{\mathrm{b}}(\boldsymbol{p})$ that is symmetric in $p_{z}$.

In the asymmetric case, characterized by the additional parameter $\Delta=\left(n_{+}-n_{-}\right) /\left(n_{+}+n_{-}\right)$with $n_{ \pm}$the densities of the two counterstreaming beams, the main effect of the asymmetry is that the range of unstable wavenumbers shrinks, with the maximum unstable wavenumber and maximum growth rate scaling as $\operatorname{Im}(\omega)_{\max }, K_{\max } \propto\left(1-\Delta^{2}\right)^{1 / 4}$ (cf. Eq. (89)) in the ultra-relativistic limit with $K c / \tilde{\omega}_{\text {bg }} \gg 1$.

In our discussion of the kinetic theory we used a mathematically convenient form of the momentum distribution: the twodimensional waterbag distribution. The results of Milosavljevic et al. (2006) gives us confidence that more realistic momentum distributions will yield qualitatively similar results. Other approaches are of course possible, such as the one taken by Tautz $\&$ Schlickeiser (2005), who employ a relativistic generalization for a drifting, two-temperature Maxwellian, where (in our notation) the case of symmetric beams is described by a momentum distribution of the form:

$f_{0 \mathrm{~b}}(\boldsymbol{p})=C \mathrm{e}^{-E / k_{\mathrm{b}} T_{\perp}}\left[\mathrm{e}^{-\alpha\left(p_{z}-p_{\mathrm{b}}\right)^{2}}+\mathrm{e}^{-\alpha\left(p_{z}+p_{\mathrm{b}}\right)^{2}}\right]$,

where $E=\sqrt{m_{\mathrm{b}}^{2} c^{4}+p_{\perp}^{2} c^{2}+p_{z}^{2} c^{2}}$, the parameter $\alpha$ is

$\alpha=\frac{1}{2 m_{\mathrm{b}} k_{\mathrm{b}}}\left(\frac{1}{T_{\|}}-\frac{1}{T_{\perp}}\right)$

and $C$ is a normalizing constant that ensures that the distribution function integrates to unity over momentum space. The quantities $T_{\|}$and $T_{\perp}$ are the temperatures for the thermal motion of the beam particles in the direction along the beam and in the plane perpendicular to the beam. We note that, by adopting the waterbag distribution function, we have assumed that there is no 
thermal motion of beam particles in the beam direction, which formally corresponds to $T_{\|}=0(\alpha=\infty)$. Recent work by Yoon (2007) suggests that the relativistic Weibel instability persists even for finite $T_{\|} \neq 0$ as long as $T_{\perp}>T_{\|}$. An important difference between our work and that of Tautz \& Schlickeiser (2005) and Yoon (2007) is the inclusion of a (hot) background plasma that reduces the growth rate at long wavelengths by screening currents, which was discussed extensively in this paper.

The beam-driven Weibel instability leads to the generation of magnetic fields over a wide range of wavelengths, as also discussed by Gruzinov \& Waxman (1999), Gruzinov (2001), Medvedev \& Loeb (1999), Frederiksen et al. (2004), and Milosavljevic et al. (2006). In this respect it behaves not unlike the original version of the instability, driven by temperature anisotropies (Weibel 1959). The next step is a detailed study of the stabilization mechanisms that terminate the phase of exponential growth of the instability discussed here. A first discussion of these stabilization mechanisms can be found in Wiersma \& Achterberg (2004). The companion paper (Achterberg et al. 2007 ) discusses them in more detail.

\section{Appendix A: Covariant formulation of plasma response}

The usual procedure for determining the response of a plasma to small-amplitude electromagnetic field, which is found in any book on plasma physics (e.g. Ichimaru 1973; Melrose 1980) relates the electric field $\boldsymbol{E}$ and the current density $\boldsymbol{J}$ it induces in the plasma by solving the linearized plasma equations. This determines the conductivity tensor $\sigma$. In the Fourier domain the amplitudes of the current density and the electric field are related by $\tilde{J}_{i}(\omega, \boldsymbol{k})=\sigma_{i j}(\omega, \boldsymbol{k}) \tilde{E}_{j}(\omega, \boldsymbol{k})$, where $(i, j)$ enumerate the spatial components and a tilde $(\sim)$ is used to denote Fourier amplitudes. One then calculates the susceptibility tensor, $\chi_{i j}(\omega, \boldsymbol{k})=\frac{4 \pi i}{\omega} \sigma_{i j}(\omega, \boldsymbol{k})$, and the dielectric tensor $\epsilon_{i j}(\omega, \boldsymbol{k})=\delta_{i j}+\chi_{i j}$ in the Fourier domain. The dispersion relation of the various linear wave modes that are supported by the plasma then follows from Maxwell's equations as $\operatorname{det}\left\{\left(c^{2} / \omega^{2}\right)\left(k_{i} k_{j}-k^{2} \delta_{i j}\right)+\epsilon_{i j}(\omega, \boldsymbol{k})\right\}=0$.

The electromagnetic response of a plasma can also be formulated covariantly (e.g. Melrose 1973, 2001; Dewar 1977). This has the advantage of immediately yielding results that are valid for an arbitrary choice of reference frame. The starting point for such a formulation is the covariant set of Maxwell's equations,

$\nabla_{\mu} F^{\mu \nu}=4 \pi J^{v}, \quad \nabla_{\mu}\left(\epsilon^{\mu v \alpha \beta} F_{\alpha \beta}\right)=0$,

with $F^{\mu v}$ the Faraday tensor defined in terms of the electromagnetic four-potential $A^{\mu} \equiv(\Phi, \boldsymbol{A})$ as (e.g. Jackson 1975)

$F^{\mu v}=\nabla^{\mu} A^{v}-\nabla^{v} A^{\mu}$,

and where $\epsilon^{\mu v \alpha \beta}$ is the totally antisymmetric (Levi-Cevita) tensor. For linear wave phenomena, this set of equations will be completed by a linear (tensorial) relation between the four-current $J^{\mu}$ and the four-potential $A^{\mu}$, see Eq. (A.4) below.

It is convenient to formulate the linear response in terms of the four-potential perturbation $\delta A^{\mu}(x)$ and the associated fourcurrent perturbation $\delta J^{\mu}(x)$. Assuming plane waves, one can represent these perturbed quantities in terms of a Fourier integral with the Fourier amplitudes $\tilde{A}^{\mu}(k)$ and $\tilde{J}^{\mu}(k)$ :

$\left(\begin{array}{l}\delta A^{\mu}(x) \\ \delta J^{\mu}(x)\end{array}\right)=\int \frac{\mathrm{d}^{4} k}{(2 \pi)^{4}}\left(\begin{array}{c}\tilde{A}^{\mu}(k) \\ \tilde{J}^{\mu}(x)\end{array}\right) \exp (-\mathrm{i} k \cdot x)$.
Here (and in what follows) a tilde $\sim$ is used to denote Fourier amplitudes of the wave-like perturbations in the various physical fields, $k^{\mu}=(\omega / c, k)$ is the wavenumber four-vector and $k \cdot x \equiv$ $k_{\mu} x^{\mu}=\omega t-\boldsymbol{k} \cdot \boldsymbol{x}$.

The Fourier-amplitude of the linearized four-current perturbation carried by some species $\mathrm{s}$ in a multi-species plasma can be related to the Fourier amplitude of the four-potential through

$4 \pi \tilde{J}_{\mathrm{s}}^{\mu}(k)=\left(\alpha_{v}^{\mu}\right)_{\mathrm{s}}(k) \tilde{A}^{v}(k)$

The quantity $\alpha_{\mathrm{s}}^{\mu v}(k)$ is the polarization tensor. The Faraday tensor associated with the waves, $\delta F^{\mu v}=\nabla^{\mu} \delta A^{v}-\nabla^{v} \delta A^{\mu}$, has the Fourier amplitude

$\tilde{F}^{\mu v}(k)=\mathrm{i}\left(k^{v} \tilde{A}^{\mu}-k^{\mu} \tilde{A}^{v}\right)$,

which follows from the replacement $\nabla^{\mu} \longrightarrow-\mathrm{i} k^{\mu}$ when going from configuration space to Fourier space. As a result, the first set of Maxwell's Eqs. (A.1) reads in the Fourier domain

$(k \cdot \tilde{A}) k^{\mu}-(k \cdot k) \tilde{A}^{\mu}=4 \pi \sum_{\mathrm{s}} \tilde{J}_{\mathrm{s}}^{\mu}=\sum_{\mathrm{s}}\left(\alpha^{\mu}{ }_{v}\right)_{\mathrm{s}} \tilde{A}^{v}$.

The polarization tensor has a number of simple properties, which are related to charge conservation and to the gauge freedom of the electromagnetic field. Charge conservation of each species $\mathrm{s}$ reads $\nabla \cdot \delta J_{\mathrm{s}}^{\mu}=0$, which in the Fourier domain becomes

$k_{\mu} \tilde{J}_{\mathrm{s}}^{\mu}(k)=0$.

Also, the four-current of each individual species should be invariant under a general gauge transformation of the form

$\delta A_{\mu} \Longrightarrow \delta A_{\mu}+\nabla_{\mu} \chi \Longleftrightarrow \tilde{A}_{\mu} \Longrightarrow \tilde{A}_{\mu}-\mathrm{i} k_{\mu} \tilde{\chi}$

Equations (A.7) and (A.8) together imply that the polarization tensor must satisfy the relations

$k_{\mu} \alpha_{\mathrm{s}}^{\mu v}(k)=\alpha_{\mathrm{s}}^{\mu v}(k) k_{v}=0$.

\section{A.1. Plasma response in the fluid approach}

If one calculates the plasma response in the fluid approximation the two conditions (A.9) imply that the linear polarization tensor of species s can always be written in the form (cf. Dewar 1977; Melrose 2001, Chap. 4):

$\left(\alpha^{\mu}{ }_{v}\right)_{\mathrm{s}}=\mathcal{P}_{\mathrm{s} \alpha}^{\mu}\left(\Pi_{\beta}^{\alpha}\right)_{\mathrm{s}} \mathcal{P}_{\mathrm{s} v}^{\dagger \beta}$

with

$\mathcal{P}_{\mathrm{s} v}^{\mu} \equiv \delta_{v}^{\mu}-\frac{U_{\mathrm{s}}^{\mu} k_{v}}{k \cdot U_{\mathrm{s}}}, \quad \mathcal{P}_{\mathrm{s} v}^{\dagger \mu} \equiv \delta_{v}^{\mu}-\frac{k^{\mu} U_{\mathrm{s} v}}{k \cdot U_{\mathrm{s}}}$

Here $U_{\mathrm{s}}^{\mu}$ is the four-velocity of the frame where species $\mathrm{s}$ is at rest, apart from the thermal motions, so that the unperturbed mass current of species $\mathrm{s}$ is $\Sigma^{\mu}=n_{\mathrm{s} 0} m_{\mathrm{s}} U_{\mathrm{s}}^{\mu}$, with $n_{\mathrm{s} 0}$ the proper density and $m_{\mathrm{s}}$ the rest mass of the species. The projection tensor $\mathcal{P}_{\mathrm{s} v}^{\mu}$ and its transpose $\mathcal{P}_{\mathrm{s} v}^{\dagger \mu}$ project to the hyperplane perpendicular to $k$, and satisfy

$k_{\mu} \mathcal{P}_{\mathrm{s} v}^{\mu}=\mathcal{P}_{\mathrm{s} v}^{\dagger \mu} k^{v}=0, \quad \mathcal{P}_{\mathrm{s} v}^{\mu} U_{\mathrm{s}}^{v}=U_{\mathrm{s} \mu} \mathcal{P}_{\mathrm{s} v}^{\dagger \mu}=0$

The determination of $\alpha_{\mathrm{s}}^{\mu \nu}$ in the fluid approach can then be reduced to the calculation of $\left(\Pi_{\beta}^{\alpha}\right)_{\mathrm{s}}$.

Linearizing the covariant fluid equation (Eq. (7) of the main paper) to first order in perturbed quantities, assuming that there 
is no electromagnetic field in the unperturbed state and that the unperturbed state is uniform and time-independent, yields:

$(\rho+e+P)_{\mathrm{s}}\left(U_{\mathrm{s}} \cdot \nabla\right) \delta U_{\mathrm{s}}^{\mu}=h_{\mathrm{s}}^{\mu v} \nabla_{v} \delta P_{\mathrm{s}}+n_{\mathrm{s}} q_{\mathrm{s}}\left(\delta F^{\mu v}\right) U_{\mathrm{s} v}$.

Here (and in what follows) $\rho_{\mathrm{s}}=n_{\mathrm{s} 0} m_{\mathrm{s}}, e_{\mathrm{s}}, P_{\mathrm{s}}$ and $U_{\mathrm{s}}^{\mu}$ are the unperturbed (equilibrium) values of the proper density, internal energy density, pressure and four-velocity of species s, and $h_{\mathrm{s}}^{\mu \nu}=$ $\eta^{\mu \nu}-U_{\mathrm{s}}^{\mu} U_{\mathrm{s}}^{\nu}$ is the projection tensor based on the unperturbed four-velocity. In the Fourier domain, using $\boldsymbol{\nabla}_{\mu} \longrightarrow-\mathrm{i} k_{\mu}$, this linearized equation of motion becomes:

$\mathrm{i}\left(k \cdot U_{\mathrm{s}}\right)(\rho+e+P)_{\mathrm{s}} \tilde{U}_{\mathrm{s}}^{\mu}=\mathrm{i} k_{\perp}^{\mu} \tilde{P}_{\mathrm{s}}-n_{\mathrm{s}} q_{\mathrm{s}} U_{\mathrm{s} v} \tilde{F}^{\mu \nu}$.

Here

$k_{\mathrm{s} \perp}^{\mu} \equiv h^{\mu v} k_{v}=k^{\mu}-\left(k \cdot U_{\mathrm{s}}\right) U_{\mathrm{s}}^{\mu}$.

The quantity $\tilde{U}_{\mathrm{s}}^{\mu}$ is the Fourier component of the four-velocity perturbation so that

$\delta U_{\mathrm{s}}^{\mu}(x)=\int \frac{\mathrm{d}^{4} k}{(2 \pi)^{4}} \tilde{U}^{\mu}(k) \exp (-\mathrm{i} k \cdot x)$.

Note that

$k \cdot U_{\mathrm{s}}=\gamma_{\mathrm{s}}\left(\omega-\boldsymbol{k} \cdot \boldsymbol{v}_{\mathrm{s}}\right)$

is a Doppler-shifted frequency that corresponds to the wave frequency in the rest frame of species s.

The continuity equation (see Eq. (8) of the main paper) upon linearization becomes

$\left(U_{\mathrm{s}} \cdot \nabla\right) \delta n_{\mathrm{s}}=-n_{\mathrm{s}}\left(\nabla \cdot \delta U_{\mathrm{s}}\right)$,

and the equation of state yields

$\delta P_{\mathrm{s}}=\Gamma_{\mathrm{s}} P_{\mathrm{s}}\left(\frac{\delta n_{\mathrm{s}}}{n_{\mathrm{s}}}\right)$

These two relations yield expressions for the density perturbation $\tilde{n}_{\mathrm{s}}$ and pressure perturbation $\tilde{P}_{\mathrm{s}}$ in the Fourier domain:

$\tilde{n}_{\mathrm{s}}=-n_{\mathrm{s}}\left(\frac{k \cdot \tilde{U}_{\mathrm{s}}}{k \cdot U_{\mathrm{s}}}\right)$.

$\tilde{P}_{\mathrm{s}}=-\Gamma_{\mathrm{s}} P_{\mathrm{s}}\left(\frac{k \cdot \tilde{U}_{\mathrm{s}}}{k \cdot U_{\mathrm{s}}}\right)$.

If one substitutes these two relations into Eq. (A.14), and substitutes relation (A.5) for the Fourier amplitude $\tilde{F}^{\mu v}$ of the Faraday tensor, one obtains a set of four linear relations between the components of the two four vectors $\tilde{U}_{\mathrm{s}}^{\mu}$ and $\tilde{A}^{v}$ :

$$
\begin{array}{r}
(\rho+e+P)_{\mathrm{s}}\left(k \cdot U_{\mathrm{s}}\right) \tilde{U}_{\mathrm{s}}^{\mu}+\Gamma_{\mathrm{s}} P_{\mathrm{s}}\left(\frac{k \cdot \tilde{U}_{\mathrm{s}}}{k \cdot U_{\mathrm{s}}}\right) k_{\mathrm{s} \perp}^{\mu} \\
=n_{\mathrm{s}} q_{\mathrm{s}}\left\{\left(U_{\mathrm{s}} \cdot \tilde{A}\right) k^{\mu}-\left(k \cdot U_{\mathrm{s}}\right) \tilde{A}^{\mu}\right\} .
\end{array}
$$

However, one of these relations is redundant as the unit normalisation of the total four-velocity implies that $\tilde{U}_{\mathrm{s}}^{\mu} \cdot U_{\mathrm{s} \mu}=0$, as is easily checked by contracting Eq. (A.21) with $U_{\mathrm{s} \mu}$.

The contribution of species $\mathrm{s}$ to the linear current fourvector is:

$\tilde{J}_{\mathrm{s}}^{\mu}=q_{\mathrm{s}} \tilde{n}_{\mathrm{s}} U_{\mathrm{s}}^{\mu}+q_{\mathrm{s}} n_{\mathrm{s}} \tilde{U}_{\mathrm{s}}^{\mu}$.

The first term in this expression is the advection current due to charge density perturbations advected by the unperturbed flow. This contribution to the current density is responsible for the occurrence of the Weibel instability. The second term is the conduction current due to the velocity perturbations induced by the electromagnetic fields. Using the first relation of Eq. (A.20), one can write the Fourier amplitude of the four-current density as

$\tilde{J}_{\mathrm{s}}^{\mu}(k)=\frac{q_{\mathrm{s}} n_{\mathrm{s}}}{k \cdot U_{\mathrm{s}}}\left\{\left(k \cdot U_{\mathrm{s}}\right) \tilde{U}_{\mathrm{s}}^{\mu}-\left(k \cdot \tilde{U}_{\mathrm{s}}\right) U_{\mathrm{s}}^{\mu}\right\}$

Solving Eq. (A.21), in effect expressing the components of $\tilde{U}_{\mathrm{s}}^{\mu}$ in terms of the components of $\tilde{A}^{\mu}$, and substituting the resulting expressions into Eq. (A.23), one can calculate the linearized current density $\tilde{J}_{\mathrm{s}}$ in terms of $\tilde{A}$, and from that read off the components of the corresponding polarization tensor $\left(\alpha_{v}^{\mu}\right)_{\mathrm{s}}$. We just give the final result in terms of the tensor $\left(\Pi_{\beta}^{\alpha}\right)_{\mathrm{s}}$ :

$\left(\Pi^{\alpha}{ }_{\beta}\right)_{\mathrm{s}}=-\tilde{\omega}_{\mathrm{ps}}^{2}\left(\delta^{\alpha}{ }_{\beta}-\frac{C_{\mathrm{s}}^{2} k^{\alpha} k_{\beta}}{\left(k \cdot U_{\mathrm{s}}\right)^{2}-K_{\mathrm{s} \perp}^{2} C_{\mathrm{s}}^{2}}\right)$.

For a cold plasma one has $C_{\mathrm{s}}^{2}=0$, so that $\left(\Pi_{\beta}^{\alpha}\right)_{\mathrm{s}}=-\tilde{\omega}_{\mathrm{ps}}^{2} \delta_{\beta}^{\alpha}$ with $\tilde{\omega}_{\mathrm{ps}}^{2}=4 \pi q_{\mathrm{s}}^{2} n_{\mathrm{s}} / m_{\mathrm{s}}$. The cold plasma result has been derived previously by Dewar (1977), his Eqs. (95) and (114).

\section{A.2. Plasma response in the kinetic approach}

In the kinetic approach one must solve the covariant Vlasov equation. Let $\mathcal{F}_{\mathrm{s}}\left(x^{\mu}, p^{\mu}\right) \mathrm{d}^{4} x \mathrm{~d}^{4} p$ be the number of particles of species $\mathrm{s}$ in the infinitesimal volume $\mathrm{d}^{4} x$ in space-time and four-momentum interval $\mathrm{d}^{4} p$. The covariant Vlasov equation is

$\frac{\mathrm{d} \mathcal{F}_{\mathrm{s}}}{\mathrm{d} \tau} \equiv \frac{\mathrm{d} x^{\mu}}{\mathrm{d} \tau} \frac{\partial \mathcal{F}_{\mathrm{s}}}{\partial x^{\mu}}+\frac{\mathrm{d} p^{\mu}}{\mathrm{d} \tau} \frac{\partial \mathcal{F}_{\mathrm{s}}}{\partial p^{\mu}}=0$

Here $\tau$ is the proper time, and

$\frac{\mathrm{d} x^{\mu}}{\mathrm{d} \tau}=u^{\mu}=\frac{p^{\mu}}{m_{\mathrm{s}}}, \quad \frac{\mathrm{d} p^{\mu}}{\mathrm{d} \tau}=q_{\mathrm{s}} F^{\mu}{ }_{\nu} u^{\nu}$

are the four-velocity and the Lorentz force acting on a particle with mass $m_{\mathrm{s}}$ and charge $q_{\mathrm{s}}$. We assume a plane-wave perturbation of the distribution function and all electromagnetic fields with the perturbations represented by a Fourier integral. The total Vlasov distribution can be written as

$\mathcal{F}(x, p)=\mathcal{F}_{0}(p)+\int \frac{\mathrm{d}^{4} k}{(2 \pi)^{4}} \tilde{\mathcal{F}}(k, p) \exp (-\mathrm{i} k \cdot x)$,

and the linearized Vlasov equation yields:

$-\mathrm{i}(k \cdot u) \tilde{\mathcal{F}}_{\mathrm{s}}(k, p)=-\frac{q_{\mathrm{s}}}{m_{\mathrm{s}}} \tilde{F}^{\mu}{ }_{v} p^{v} \frac{\partial \mathcal{F}_{0 \mathrm{~s}}(p)}{\partial p^{\mu}}$.

Here we have assumed that the unperturbed distribution $\mathcal{F}_{0}(p)$ is uniform in space-time and that there is no unperturbed electromagnetic field. The linear current density associated with this perturbation has an amplitude

$\tilde{J}_{\mathrm{s}}^{\mu}(k)=\frac{q_{\mathrm{s}}}{m_{\mathrm{s}}} \int \mathrm{d}^{4} p p^{\mu} \tilde{\mathcal{F}}_{\mathrm{s}}(k, p)$

for particle species s. Using Eq. (A.5) for the Faraday tensor $\tilde{F}_{v}^{\mu}$ and solving (A.28) for $\tilde{\mathcal{F}}(k, p)$ and substituting the resulting expression one finds:

$4 \pi \tilde{J}_{\mathrm{s}}^{\mu}=\frac{4 \pi q_{\mathrm{s}}^{2}}{m_{\mathrm{s}}} \int \mathrm{d}^{4} p p^{\mu}\left(\tilde{A}^{v}-\frac{(\tilde{A} \cdot p)}{(k \cdot p)} k^{\nu}\right) \frac{\partial \mathcal{F}_{0 \mathrm{~s}}(p)}{\partial p^{v}}$ 
After a partial integration one can write (A.30) in the form $4 \pi \tilde{J}_{\mathrm{s}}^{\mu}=\left(\alpha_{v}^{\mu}\right)_{\mathrm{s}} \tilde{A}^{v}$, which defines the polarization tensor of species $\mathrm{s}$ in the kinetic approach:

$\left(\alpha_{v}^{\mu}\right)_{\mathrm{s}} \equiv-\frac{4 \pi q_{\mathrm{s}}^{2}}{m_{\mathrm{s}}} \int \mathrm{d}^{4} p \mathcal{F}_{0 \mathrm{~s}}(p) M_{v}^{\mu}(k, p)$,

with

$M_{v}^{\mu}(k, p)=\delta_{v}^{\mu}-\frac{k^{\mu} p_{v}+p^{\mu} k_{v}}{(k \cdot p)}+\frac{(k \cdot k) p^{\mu} p_{v}}{(k \cdot p)^{2}}$.

This result is in fact closely related to the fluid results (A.10) and (A.11). This can be seen by writing (A.31) in the form

$\left(\alpha_{v}^{\mu}\right)_{\mathrm{s}}(k)=\int \mathrm{d}^{4} p \mathcal{F}_{0 \mathrm{~s}}(p)\left[\mathcal{P}_{\alpha}^{\mu}(k, p)\left(\Pi_{\beta}^{\alpha}\right)_{\mathrm{s}} \mathcal{P}_{v}^{\dagger \beta}(k, p)\right]$,

with

$\mathcal{P}_{\alpha}^{\mu}(k, p)=\delta_{\alpha}^{\mu}-\frac{p^{\mu} k_{\alpha}}{(k \cdot p)}$

$\mathcal{P}^{\dagger \beta}(k, p)=\delta_{v}^{\beta}-\frac{k^{\beta} p_{v}}{(k \cdot p)}$

and

$\left(\Pi_{\beta}^{\alpha}\right)_{\mathrm{s}}=-\frac{4 \pi q_{\mathrm{s}}^{2}}{m_{\mathrm{s}}} \delta_{\beta}^{\alpha}$.

In the case of cold beams the fluid and kinetic results are completely equivalent.

Result (A.31) was derived in a different context by Achterberg (1986), and also corresponds to expression 227 of Dewar (1977) and the corresponding expression in Melrose (2001), Chap. 4.1, for the response tensor of an unmagnetized plasma. These relations show explicitly that both charge conservation, and the invariance of the current density under gauge transformations of the vector potential, are once again ensured.

\section{Appendix B: Covariant dispersion relation}

The linear dispersion relation for modes supported by the plasma follows from Eq. (A.6), which can be written as

$D^{\mu v}(k) \tilde{A}_{v}(k)=0$.

Here we define

$D^{\mu \nu}(k) \equiv(k \cdot k) \eta^{\mu \nu}-k^{\mu} k^{\nu}+\alpha^{\mu \nu}(k)$

with $\alpha^{\mu v}(k) \equiv \sum_{\mathrm{s}}\left(\alpha^{\mu v}\right)_{\mathrm{s}}(k)$. However, this set of equations is not linearly independent. The tensor $D^{\mu v}$ satisfies (see Eq. (A.9)): $k_{\mu} D^{\mu \nu}=D^{\mu \nu} k_{v}=0$. This means that the determinant of the $4 \times 4$ matrix $D^{\mu \nu}$ vanishes identically: $\operatorname{det}\left[D^{\mu \nu}\right]=0$.

The dispersion relation for linear waves can be obtained by noting that one can factor Eq. (B.1) as:

$\left(\delta_{\alpha}^{\mu}-\frac{k^{\mu} k_{\alpha}}{(k \cdot k)}\right)\left[(k \cdot k) \eta^{\alpha \nu}+\alpha^{\alpha \nu}\right] \tilde{A}_{v}=0$,

since $k_{\alpha} \alpha^{\alpha v}=0$. The first factor leads to the vanishing determinant of $D^{\mu v}$. Therefore, the dispersion relation for the waves in the plasma follows from the requirement that the determinant of the second factor vanishes identically:

$\operatorname{det}\left[(k \cdot k) \eta^{\mu v}+\sum_{\mathrm{s}} \alpha_{\mathrm{s}}^{\mu v}(k)\right]=0$.
The problem of determining the wave properties therefore corresponds to determining the polarization tensor $\alpha^{\mu \nu}$ and solving the dispersion relation (B.4)

In this paper we take a different (but equivalent) approach, which avoids dealing with the problem $\operatorname{det}\left[D^{\mu \nu}\right]=0$ altogether. First of all, we impose the invariant Lorentz-gauge on the wave electromagnetic fields,

$k^{\mu} \tilde{A}_{\mu}(k)=0$.

This choice is convenient, but it should be stressed that the correct dispersion relation can be derived in any gauge. The one longitudinal and two transverse degrees of freedom in a plasma can be described by three "unit" polarization vectors $e_{\ell}^{\mu}, e_{\mathrm{tr} 1}^{\mu}$ and $e_{\mathrm{tr} 2}^{\mu}$. In addition, one has the gauge degree of freedom (Eq. (A.8)) which defines a fourth gauge vector: $e_{\mathrm{G}}^{\mu} \equiv k^{\mu} / \sqrt{|k \cdot k|}$. We will not need to consider the degenerate case where $k \cdot k=0$.

These four vectors define an orthonormal tetrad $e_{i}$ with $e_{0}^{\mu}=$ $e_{\mathrm{G}}^{\mu}, e_{1}^{\mu}=e_{\ell}^{\mu}, e_{2}^{\mu}=e_{\mathrm{tr} 1}^{\mu}$ and $e_{3}^{\mu}=e_{\mathrm{tr} 2}^{\mu}$. This tetrad can be used to describe all electromagnetic wave-like phenomena in a plasma. Assuming that the ordinary wave vector $\boldsymbol{k}$ is along the $x$-axis so that $k^{\mu}=(\omega, K, 0,0)$, we can choose these four vectors as ${ }^{2}$

$e_{\mathrm{G}}^{\mu}=\frac{(\omega, K, 0,0)}{\sqrt{|k \cdot k|}}, e_{\ell}^{\mu}=\frac{(K, \omega, 0,0)}{\sqrt{|k \cdot k|}}$,

$e_{\mathrm{tr} 1}^{\mu}=(0,0,1,0), e_{\mathrm{tr} 2}^{\mu}=(0,0,0,1)$.

The three polarization vectors satisfy

$k \cdot e_{\ell}=k \cdot e_{\mathrm{tr} 1}=k \cdot e_{\mathrm{tr} 2}=0$.

Using these definitions one has

$e_{i} \cdot e_{j} \equiv g_{i j}=\operatorname{diag}( \pm 1, \mp 1,-1,-1)$,

where the upper sign applies in the super-luminal case, $\omega^{2}-K^{2}>$ 0 , and the lower sign in the sub-luminal case, $\omega^{2}-K^{2}<0$. This choice of polarization vectors is convenient for the problem at hand, but depends on our adoption of the Lorentz gauge.

Adoption of the Lorentz gauge implies that one can expand the four-potential in terms of the three polarization vectors as

$\tilde{A}^{\mu}(k)=\sum_{j=1}^{3} \tilde{\mathcal{A}}^{j}(k) e_{j}^{\mu}$.

This defines three independent four-potential components ${ }^{3}$. Contracting the wave Eq. (B.1) from the left with $e_{i}^{\mu}$ one finds, using relation (B.7) and definition (B.8), that the wave equation can be written as

$\mathcal{D}_{i j}(k) \tilde{\mathcal{A}}^{j}(k)=0$.

Here we employ the summation convention for $i, j=1,2,3$ and define the $3 \times 3$ dispersion matrix $\mathcal{D}_{i j}$ by

$\mathcal{D}_{i j}(k) \equiv e_{i}^{\mu} D_{\mu \nu} e_{j}^{\nu}=(k \cdot k) g_{i j}+\alpha_{i j}$,

where

$\alpha_{i j}(k) \equiv \sum_{s} e_{i}^{\mu}\left(\alpha_{\mu v}\right)_{s} e_{j}^{v} \equiv \sum_{s}\left(\alpha_{i j}\right)_{s}(k)$.

${ }^{2}$ We limit the discussion to the case where $k \cdot k=\omega^{2}-K^{2}$ is a real quantity, which includes the case of a purely imaginary frequency where $\omega^{2}<0$.

3 The Lorentz-gauge implies $\mathcal{A}_{0}=e_{0} \cdot A=0$. 
The gauge vector $e_{\mathrm{G}}$ does not figure in these equations. Since $k_{\mu} D^{\mu v}=D^{\mu v} k_{v}=0$, the result of gauge invariance and charge conservation, the component $\mathcal{D}_{00}$ vanishes identically, as do $\mathcal{D}_{0 i}$ and $\mathcal{D}_{i 0}$, where $i=1,2,3$. This is entirely the result of our choice of the Lorentz gauge, and in particular of our adoption of $e_{0}^{\mu}=k^{\mu} / \sqrt{|k \cdot k|}$.

This procedure correctly isolates the physical wave modes involving the three vectors $e_{\ell}$ and $e_{\mathrm{tr} 1,2}$, and removes the apparent singularity in the calculation of the determinant of $D^{\mu \nu}$. The dispersion relation for electromagnetic waves in a plasma therefore follows from the solution condition for (B.10):

$\operatorname{det}\left[\mathcal{D}_{i j}(k)\right]=0$.

Another choice of gauge corresponds to another choice of the polarization vectors $e_{i}$, and of the components of $\mathcal{D}_{i j}$. However, the resulting dispersion relation, again formally given by (B.13), will yield the same modes.

\section{Appendix C: The components of the dispersion tensor $\mathcal{D}_{\mathrm{ij}}$}

For the sake of completeness we give all the non-zero components of the dispersion tensor used in this paper, assuming a wave vector $\boldsymbol{k}=K \hat{\boldsymbol{x}}$ and wave frequency $\omega$ in the lab frame, where the background plasma is at rest.

\section{C.1. Fluid approximation}

In addition to the background plasma with sound speed $C_{\mathrm{s}}=$ $\Gamma P_{\mathrm{s}} /(\rho+e+P)_{\mathrm{s}}$ for species $\mathrm{s}$, there are two beams with bulk four-velocity $U_{\mathrm{b}}^{\mu}=\gamma_{\mathrm{b}}\left(1,0,0, \pm V_{\mathrm{b}}\right)$, and a proper density

$n_{ \pm}=\frac{1 \pm \Delta}{2} n_{\mathrm{b}}$

Here $n_{\mathrm{b}}$ is the total proper density of the two beams. For simplicity we assume that the two beams have equal temperature so that their internal sound speed satisfies $C_{+}=C_{-}=C_{\mathrm{b}}$.

For waves propagating in the direction perpendicular to the beam so that $k^{\mu}=(\omega, K, 0,0)$ the components of the dispersion tensor are:

$\mathcal{D}_{11}=-|k \cdot k|\left(1-\sum_{s \in \mathrm{bg}} \frac{\tilde{\omega}_{\mathrm{ps}}^{2}}{\omega^{2}-K^{2} C_{\mathrm{s}}^{2}}-\frac{\tilde{\omega}_{\mathrm{pb}}^{2}}{\omega^{2}-K^{2} \tilde{C}_{\mathrm{b}}^{2}}\right)$,
$\mathcal{D}_{22}=K^{2}+\sum_{s \in \mathrm{bg}} \tilde{\omega}_{\mathrm{ps}}^{2}+\tilde{\omega}_{\mathrm{pb}}^{2}-\omega^{2}$,
$\mathcal{D}_{33}=K^{2}+\sum_{s \in \mathrm{bg}} \tilde{\omega}_{\mathrm{ps}}^{2}+\tilde{\omega}_{\mathrm{pb}}^{2}\left(1-\frac{\left(\omega^{2}-K^{2}\right) \tilde{V}_{\mathrm{b}}^{2}}{\omega^{2}-K^{2} \tilde{C}_{\mathrm{b}}^{2}}\right)-\omega^{2}$,
$\mathcal{D}_{31}=\Delta(\sqrt{|k \cdot k|})\left[\frac{\tilde{\omega}_{\mathrm{pb}}^{2}}{\gamma_{\mathrm{C}_{\mathrm{b}}} \sqrt{1-C_{\mathrm{b}}^{2} V_{\mathrm{b}}^{2}}}\left(\frac{K \tilde{V}_{\mathrm{b}}}{\omega^{2}-K^{2} \tilde{C}_{\mathrm{b}}^{2}}\right)\right]$.

Here we have defined

$\tilde{C}_{\mathrm{b}}=\frac{C_{\mathrm{b}}}{\gamma_{\mathrm{b}} \sqrt{1-C_{\mathrm{b}}^{2} V_{\mathrm{b}}^{2}}}, \quad \tilde{V}_{\mathrm{b}}=\frac{V_{\mathrm{b}}}{\gamma_{\mathrm{C}_{\mathrm{b}}} \sqrt{1-C_{\mathrm{b}}^{2} V_{\mathrm{b}}^{2}}}$

with $\gamma_{C_{\mathrm{b}}} \equiv 1 / \sqrt{1-C_{\mathrm{b}}^{2}}$, and

$\tilde{\omega}_{\mathrm{ps}}^{2} \equiv \frac{4 \pi n_{\mathrm{s}}^{2} q_{\mathrm{s}}^{2}}{(\rho+e+P)_{\mathrm{s}}}, \quad \tilde{\omega}_{\mathrm{pb}}^{2}=\frac{4 \pi q_{\mathrm{b}}^{2} n_{\mathrm{b}}}{m_{\mathrm{b}} h_{\mathrm{b}}}$.
The beam enthalpy per unit mass that appears in the expression for $\tilde{\omega}_{\mathrm{pb}}$ equals $h_{\mathrm{b}}=1+(e+P)_{ \pm} / n_{ \pm} m_{\mathrm{b}}$, and is identical for the two beams.

\section{C.2. Kinetic theory}

We express the covariant distribution $\mathcal{F}_{0}$ in terms of the more familiar phase-space density $f_{0 \mathrm{~s}}(\boldsymbol{x}, t, \boldsymbol{p})$, which is defined in terms of the ordinary momentum $\boldsymbol{p}$ and particle density $\bar{n}_{\mathrm{s}}$ in the lab frame. Particles must lie on the mass shell: $\left(p^{0}\right)^{2}-|\boldsymbol{p}|^{2}=$ $E^{2}-|p|^{2}=m_{\mathrm{s}}^{2}$ for $c=1$. If one assumes that the unperturbed distribution is spatially uniform and time-independent, the relation between $\mathcal{F}_{0 \mathrm{~s}}$ and $f_{0 \mathrm{~s}}$ reads

$\mathcal{F}_{0 \mathrm{~s}}\left(x^{\mu}, p^{\mu}\right)=\bar{n}_{\mathrm{s}} f_{0 \mathrm{~s}}(\boldsymbol{p}) \frac{\delta\left(p^{0}-\gamma(\boldsymbol{p}) m_{\mathrm{s}}\right)}{\gamma(\boldsymbol{p})}$

Here $\gamma(\boldsymbol{p})=\sqrt{1+|\boldsymbol{p}|^{2} / m_{\mathrm{s}}^{2}}$. The momentum distribution $f_{0 \mathrm{~s}}(\boldsymbol{p})$ has been normalized so that

$\int \mathrm{d}^{3} \boldsymbol{p} f_{0 \mathrm{~s}}(\boldsymbol{p})=1$

The quantity $\bar{n}_{\mathrm{s}}$ is the lab frame density of species s. The momentum integration then formally changes according to $\mathrm{d}^{4} p F_{0 \mathrm{~s}}\left(p^{\mu}\right) \Longrightarrow \bar{n}_{\mathrm{s}} \mathrm{d}^{3} \boldsymbol{p} f_{0 \mathrm{~s}}(\boldsymbol{p}) / \gamma(\boldsymbol{p})$, which remains Lorentzinvariant as both $\mathrm{d}^{3} \boldsymbol{p} / \gamma(\boldsymbol{p})$ and $\bar{n}_{\mathrm{s}} f_{0 \mathrm{~s}}$ are Lorentz-invariants (e.g. Landau \& Lifshitz 1975).

As in the fluid treatment, we can use the set of polarization vectors and their properties to derive the kinetic equivalent of the fluid results. General expression (A.31) for the covariant polarization tensor $\left(\alpha_{v}^{\mu}\right)$, together with the choice (B.6) for the polarization vectors, leads to the following components of the corresponding polarization tensor $\left(\alpha_{i j}\right)_{\mathrm{s}}$ of particle species $\mathrm{s}$ in the Lorentz-gauge $k_{\mu} \tilde{A}^{\mu}=0$ :

$\left(\alpha_{i j}\right)_{\mathrm{s}}=-\frac{4 \pi q_{\mathrm{s}}^{2} \bar{n}_{\mathrm{s}}}{m_{\mathrm{s}}} \int \frac{\mathrm{d}^{3} \boldsymbol{p}}{\gamma(\boldsymbol{p})} f_{0 \mathrm{~s}}(\boldsymbol{p}) \mathcal{M}_{i j}(k, p)$.

Here $\mathcal{M}_{i j}(k, p)$ is defined as

$\mathcal{M}_{i j} \equiv e_{i}^{\mu} M_{\mu v} e_{j}^{v}=g_{i j}+\frac{(k \cdot k) p_{i} p_{j}}{(k \cdot p)^{2}}$,

with $p_{i} \equiv p \cdot e_{i}$, where $p^{\mu}=\left(\gamma(\boldsymbol{p}) m_{\mathrm{s}}, \boldsymbol{p}\right)$.

The components of the polarization tensor with $k^{\mu}=$ $(\omega, K, 0,0)$ follow as

$$
\begin{aligned}
\alpha_{11}^{\mathrm{b}} & =|k \cdot k| \frac{4 \pi q_{\mathrm{b}}^{2} \bar{n}_{\mathrm{b}}}{m_{\mathrm{b}}} \int \frac{\mathrm{d}^{3} \boldsymbol{p}}{\gamma(\boldsymbol{p})} f_{0 \mathrm{~b}}(\boldsymbol{p})\left\{\frac{1-v_{x}^{2}}{\left(\omega-K v_{x}\right)^{2}}\right\}, \\
\alpha_{22}^{\mathrm{b}} & =\frac{4 \pi q_{\mathrm{b}}^{2} \bar{n}_{\mathrm{b}}}{m_{\mathrm{b}}} \int \frac{\mathrm{d}^{3} \boldsymbol{p}}{\gamma(\boldsymbol{p})} f_{0 \mathrm{~b}}(\boldsymbol{p}), \\
\alpha_{33}^{\mathrm{b}} & =\frac{4 \pi q_{\mathrm{b}}^{2} \bar{n}_{\mathrm{b}}}{m_{\mathrm{b}}} \int \frac{\mathrm{d}^{3} \boldsymbol{p}}{\gamma(\boldsymbol{p})} f_{0 \mathrm{~b}}(\boldsymbol{p})\left\{1-\frac{v_{z}^{2}\left(\omega^{2}-K^{2}\right)}{\left(\omega-K v_{x}\right)^{2}}\right\}, \\
\alpha_{31}^{\mathrm{b}} & =\alpha_{13}^{\mathrm{b} *} \\
& =-\sqrt{|k \cdot k|} \frac{4 \pi q_{\mathrm{b}}^{2} \bar{n}_{\mathrm{b}}}{m_{\mathrm{b}}} \int \frac{\mathrm{d}^{3} \boldsymbol{p}}{\gamma(\boldsymbol{p})} f_{0 \mathrm{~b}}(\boldsymbol{p})\left\{\frac{v_{z}\left(K-\omega v_{x}\right)}{\left(\omega-K v_{x}\right)^{2}}\right\} .
\end{aligned}
$$

Here $\boldsymbol{v}=\boldsymbol{p} / \gamma m_{\mathrm{b}}$ is the velocity of beam particles with momentum $p$. 
It can be shown by partial integration that this is equivalent with

$$
\begin{aligned}
\alpha_{11}^{\mathrm{b}}= & -\frac{4 \pi q_{\mathrm{b}}^{2} \bar{n}_{\mathrm{b}}|k \cdot k|}{K^{2}} \int \mathrm{d}^{3} \boldsymbol{p}\left\{\frac{K \frac{\partial f_{0 \mathrm{~b}}(\boldsymbol{p})}{\partial p_{x}}}{\omega-K v_{x}}\right\}, \\
\alpha_{33}^{\mathrm{b}}= & \frac{4 \pi q_{\mathrm{b}}^{2} \bar{n}_{\mathrm{b}}}{m_{\mathrm{b}}} \int \frac{\mathrm{d}^{3} \boldsymbol{p}}{\gamma(\boldsymbol{p})} f_{0 \mathrm{~b}}(\boldsymbol{p})\left(1-v_{z}^{2}\right) \\
& -4 \pi q_{\mathrm{b}}^{2} \bar{n}_{\mathrm{b}} \int \mathrm{d}^{3} \boldsymbol{p}\left\{\frac{v_{z}^{2}}{\omega-K v_{x}}\left(K \frac{\partial f_{0 \mathrm{~b}}(\boldsymbol{p})}{\partial p_{x}}\right)\right\}, \\
\alpha_{31}^{\mathrm{b}}= & \frac{\sqrt{|k \cdot k|}}{\omega}\left[\frac{4 \pi q_{\mathrm{b}}^{2} \bar{n}_{\mathrm{b}}}{m_{\mathrm{b}}} \int \frac{\mathrm{d}^{3} \boldsymbol{p}}{\gamma(\boldsymbol{p})} f_{0 \mathrm{~b}}(\boldsymbol{p}) v_{x} v_{z}\right. \\
& \left.+4 \pi q_{\mathrm{b}}^{2} \bar{n}_{\mathrm{b}} \int \mathrm{d}^{3} \boldsymbol{p}\left\{\frac{v_{x} v_{z}}{\omega-K v_{x}}\left(K \frac{\partial f_{0 \mathrm{~b}}(\boldsymbol{p})}{\partial p_{x}}\right)\right\}\right] .
\end{aligned}
$$

This particular form is convenient in the waterbag approximation discussed below.

\section{C.3. Waterbag approximation}

We use a momentum distribution for the beams of the form

$$
\begin{aligned}
f_{0 \mathrm{~b}}(\boldsymbol{p})= & \frac{\Theta\left(p_{x}+p_{x 0}\right)-\Theta\left(p_{x}-p_{x 0}\right)}{2 p_{x 0}} \delta\left(p_{y}\right) \\
& \times\left[\frac{1+\Delta}{2} \delta\left(p_{z}-p_{z 0}\right)+\frac{1-\Delta}{2} \delta\left(p_{z}+p_{z 0}\right)\right] .
\end{aligned}
$$

For the background we continue to use the fluid approximation.

Substituting (C.11) into (C.10) the evaluation of the $\alpha_{i j}^{\mathrm{b}}$ is straightforward as the $p_{x}$-derivatives in these expressions convert the Heaviside step-functions in (C.11) into Dirac deltafunctions. The resulting components of the dispersion tensor are:

$$
\begin{aligned}
& \mathcal{D}_{11}=-|k \cdot k|\left(1-\sum_{s \in \mathrm{bg}} \frac{\tilde{\omega}_{\mathrm{ps}}^{2}}{\omega^{2}-K^{2} C_{\mathrm{s}}^{2}}-\frac{\hat{\omega}_{\mathrm{pb}}^{2}}{\omega^{2}-K^{2} V_{x 0}^{2}}\right), \\
& \mathcal{D}_{22}=K^{2}+\sum_{s \in \mathrm{bg}} \tilde{\omega}_{\mathrm{ps}}^{2}+\hat{\omega}_{\mathrm{pb}}^{2} \tilde{\mathcal{G}}_{\mathrm{b}}-\omega^{2}, \\
& \mathcal{D}_{33}=K^{2}+\sum_{s \in \mathrm{bg}} \tilde{\omega}_{\mathrm{ps}}^{2}+\hat{\omega}_{\mathrm{pb}}^{2}\left(\mathcal{G}_{\mathrm{b}}+\frac{K^{2} V_{z 0}^{2}}{\omega^{2}-K^{2} V_{x 0}^{2}}\right)-\omega^{2}, \\
& \mathcal{D}_{31}=\Delta(\sqrt{|k \cdot k|})\left[\frac{\hat{\omega}_{\mathrm{pb}}^{2} K V_{z 0}}{\omega^{2}-K^{2} V_{x 0}^{2}}\right] .
\end{aligned}
$$

Here we define the beam plasma frequency by

$$
\hat{\omega}_{\mathrm{pb}}^{2}=\frac{4 \pi q_{\mathrm{b}}^{2} \bar{n}_{\mathrm{b}}}{\gamma_{0} m_{\mathrm{b}}},
$$

define a characteristic Lorentz-factor and velocity components

$$
\begin{aligned}
& \gamma_{0}=\sqrt{1+\frac{p_{x 0}^{2}}{m_{\mathrm{b}}^{2} c^{2}}+\frac{p_{z 0}^{2}}{m_{\mathrm{b}}^{2} c^{2}}}, \\
& V_{x 0}=\frac{p_{x 0}}{\gamma_{0} m_{\mathrm{b}}}, \quad V_{z 0}=\frac{p_{z 0}}{\gamma_{0} m_{\mathrm{b}}},
\end{aligned}
$$

and introduce the two functions

$$
\begin{aligned}
\tilde{\mathcal{G}}_{\mathrm{b}} & =\gamma_{0} \int \frac{\mathrm{d}^{3} \boldsymbol{p}}{\gamma(\boldsymbol{p})} f_{0 \mathrm{~b}}(\boldsymbol{p})=\frac{1}{2 V_{x 0}} \ln \left[\frac{1+V_{x 0}}{1-V_{x 0}}\right] \\
\mathcal{G}_{\mathrm{b}} & =\gamma_{0} \int \frac{\mathrm{d}^{3} \boldsymbol{p}}{\gamma(\boldsymbol{p})} f_{0 \mathrm{~b}}(\boldsymbol{p})\left(1-v_{z}^{2}\right) \\
& =\tilde{\mathcal{G}}_{\mathrm{b}}-\frac{p_{z 0}^{2}}{p_{z 0}^{2}+m_{\mathrm{b}}^{2} c^{2}} .
\end{aligned}
$$

The result for the beam contribution to the polarization tensor, $\alpha_{i j}^{\mathrm{b}}$, has been derived by Silva et al. (2002), using a somewhat different notation.

\section{Appendix D: Magnetized Weibel instability}

We briefly consider the magnetized case, in order to compare the results obtained with the present formalism with those of Tautz \& Schlickeiser (2006). We limit the discussion to the case where the electric field vanishes in the laboratory frame (the rest frame of the background plasma), and where the beam velocity is along the magnetic field in the $z$-direction. The beam trajectory then remains straight, and we do not have to deal with the much more difficult case of oscillator beams that gyrate around the ambient magnetic field. This means that the Lorentz force on all components vanishes in the unperturbed state: in a covariant notation

$F^{\mu v} U_{\mathrm{s} v}=0$.

In both the laboratory frame and the rest frame of the beams, moving with velocity $\pm V_{\mathrm{b}}$ along the $z$-axis, the only nonvanishing components of the Faraday tensor are $F^{21}=-F^{12}=$ $B$, with $B$ the strength of the magnetic field $\boldsymbol{B}=B \hat{z}$ in the laboratory frame.

In the fluid approximation the linearized equation of motion for small perturbations in the four-velocity of species s now reads, when transformed to the Fourier domain:

$$
\begin{aligned}
(\rho+e+P)_{\mathrm{s}}\left(k \cdot U_{\mathrm{s}}\right) \tilde{U}_{\mathrm{s}}^{\mu} & +\Gamma_{\mathrm{s}} P_{\mathrm{s}}\left(\frac{k \cdot \tilde{U}_{\mathrm{s}}}{k \cdot U_{\mathrm{s}}}\right) k_{\mathrm{s} \perp}^{\mu}-i n_{\mathrm{s}} q_{\mathrm{s}} F^{\mu v} \tilde{U}_{\mathrm{s} \mu} \\
& =n_{\mathrm{s}} q_{\mathrm{s}}\left\{\left(U_{\mathrm{s}} \cdot \tilde{A}\right) k^{\mu}-\left(k \cdot U_{\mathrm{s}}\right) \tilde{A}^{\mu}\right\} .
\end{aligned}
$$

The last term on the left-hand side gives the first-order Lorentz force. Solving this equation, expressing the four-velocity perturbation in terms of the vector potential $\tilde{A}^{\mu}$, and calculating the associated four-current density, one finds the polarization tensor $\alpha_{v}^{\mu}$. As before, we express the final result in terms of the tensor $\left(\Pi_{\beta}^{\alpha}\right)_{\mathrm{s}}$ for each species in the plasma:

$$
\begin{aligned}
\left(\Pi_{\beta}^{\alpha}\right)_{\mathrm{s}}= & -\frac{\tilde{\omega}_{\mathrm{ps}}^{2}}{N(k)}\left\{\left(k \cdot U_{\mathrm{s}}\right)^{2}\left[\left(k \cdot U_{\mathrm{s}}\right)^{2}-K_{\mathrm{s} \perp}^{2} C_{\mathrm{s}}^{2}\right] \delta_{\beta}^{\alpha}\right. \\
& -i\left(k \cdot U_{\mathrm{s}}\right) \Omega_{\mathrm{s}}\left[\left(k \cdot U_{\mathrm{s}}\right)^{2}-(k \cdot b)^{2} C_{\mathrm{s}}^{2}\right] \epsilon_{\beta \mu \nu}^{\alpha} U_{\mathrm{s}}^{\mu} b^{v} \\
& +i\left(k \cdot U_{\mathrm{s}}\right) \Omega_{\mathrm{s}}(k \cdot b) C_{\mathrm{s}}^{2} k^{v} U_{\mathrm{s}}^{\mu} b^{\lambda}\left[\epsilon_{\nu \mu \lambda}^{\alpha} b_{\beta}-b^{\alpha} \epsilon_{\beta \nu \mu \lambda}\right] \\
& \left.-\left(k \cdot U_{\mathrm{s}}\right)^{2} C_{\mathrm{s}}^{2} k^{\alpha} k_{\beta}+\left(k \cdot U_{\mathrm{s}}\right)^{2} \Omega_{\mathrm{s}}^{2} b^{\alpha} b_{\beta}\right\}
\end{aligned}
$$

In this expression we define

$$
\begin{aligned}
N(k) \equiv & \left(k \cdot U_{\mathrm{s}}\right)^{2}\left[\left(k \cdot U_{\mathrm{s}}\right)^{2}-K_{\mathrm{s} \perp}^{2} C_{\mathrm{s}}^{2}\right] \\
& -\Omega_{\mathrm{s}}^{2}\left[\left(k \cdot U_{\mathrm{s}}\right)^{2}-(k \cdot b)^{2} C_{\mathrm{s}}^{2}\right]
\end{aligned}
$$


and

$\Omega_{\mathrm{s}} \equiv \frac{n_{\mathrm{s}} q_{\mathrm{s}} B}{(\rho+e+P)_{\mathrm{s}}}$,

which is an effective gyrofrequency with $B$ the magnetic field strength in the lab frame. In this particular case it is also the strength of the magnetic field in the rest frame of the beams, as follows from the Lorentz-transformations of the electromagnetic field, e.g. Jackson (1975), Chap. 11. The quantity $K_{\mathrm{s} \perp}^{2}$ is defined in Eq. (11) of the main paper. The quantity $\epsilon^{\alpha \beta \gamma \delta}$ is the totally antisymmetric Levi-Cevita tensor, and the four-vector $b^{\mu}=(0, \boldsymbol{B} / B)$ corresponds to a space-like vector whose spatial component is along the magnetic field in the lab frame, where it takes the form $b^{\mu}=(0,0,0,1)$. It is easily checked that we recover the earlier result (A.23) for the unmagnetized case by putting $\Omega_{\mathrm{s}}=0$.

We limit the discussion to the case where the wavevector $\boldsymbol{k}=$ $K \hat{\boldsymbol{x}}$ in the laboratory frame is perpendicular to the magnetic field $\boldsymbol{B}=B \hat{z}$ (and the beam velocity) so that $(k \cdot b)=0$. We assume two symmetric counterstreaming beams. The Weibel instability then corresponds to the instability of the ordinary mode, cf. Tautz \& Schlickeiser (2006), with the wave electric field $\delta \boldsymbol{E}$ along the unperturbed magnetic field in the laboratory frame where the background plasma is at rest. A straightforward calculation then shows that the relevant component of the dispersion tensor, $\mathcal{D}_{33}$, becomes:

$\mathcal{D}_{33} \equiv K^{2}-\omega^{2}+\sum_{s \in \mathrm{bg}} \tilde{\omega}_{\mathrm{ps}}^{2}+\tilde{\omega}_{\mathrm{pb}}^{2}\left(1-\frac{\left(\omega^{2}-K^{2}\right) \tilde{V}_{\mathrm{b}}^{2}}{\omega^{2}-K^{2} \tilde{C}_{\mathrm{b}}^{2}-\tilde{\Omega}_{\mathrm{b}}^{2}}\right)$.

Here we define

$\tilde{\Omega}_{\mathrm{b}}=\frac{\Omega_{\mathrm{b}}}{\gamma_{\mathrm{b}} \sqrt{1-V_{\mathrm{b}}^{2} C_{\mathrm{b}}^{2}}}$

and all other quantities have been defined in Appendix C, Eqs. (C.3) and (C.4).

The Weibel dispersion relation, $\mathcal{D}_{33}=0$, again takes the form of a bi-quadratic equation for the lab-frame wave frequency $\omega$, see Eq. (34) of the main paper, but now with the coefficients

$\mathcal{B}(K)=\tilde{\omega}_{\mathrm{bg}}^{2}+\tilde{\Omega}_{\mathrm{b}}^{2}+\tilde{\omega}_{\mathrm{pb}}^{2}\left(1-\tilde{V}_{\mathrm{b}}^{2}\right)+K^{2}\left(1+\tilde{C}_{\mathrm{b}}^{2}\right)$,

and

$C(K)=\left(\tilde{\omega}_{\mathrm{bg}}^{2}+\tilde{\omega}_{\mathrm{pb}}^{2}+K^{2}\right)\left(K^{2} \tilde{C}_{\mathrm{b}}^{2}+\tilde{\Omega}_{\mathrm{b}}^{2}\right)-\tilde{\omega}_{\mathrm{pb}}^{2} K^{2} \tilde{V}_{\mathrm{b}}^{2}$.
This corresponds to the replacement $K^{2} \tilde{C}_{\mathrm{b}}^{2} \longrightarrow K^{2} \tilde{C}_{\mathrm{b}}^{2}+\tilde{\Omega}_{\mathrm{b}}^{2}$ with respect to the field-free case. As before, the instability condition reads $C(K)<0$.

\section{References}

Abramowitz, M., \& Stegun, I. A. 1970, Handbook of Mathematical Functions (New York: Dover Publications), 80

Achterberg, A. 1986, J. Plasma Phys., 35, 257

Achterberg, A., Wiersma, J., \& Norman, C. A. 2007, A\&A, 475, 19

Akhiezer, A. I., Akhiezer, I. A., Polovin, R. V., Sitenko, A. G., \& Stepanov, K. N. 1975, Plasma Electrodynamics Volume 1: Linear Theory (Oxford: Pergamon Press)

Alexandrov, A. F., Bogdankevich, L. S., \& Rukhadze, A. A. 1984, Principles of Plasma Electrodynamics (Springer Verlag), 105

Blandford, R. D., \& McKee, C. F. 1976, Phys. Fl., 19, 1130

Califano, F., Cecchi, T., \& Chiuderi, C. 2002, Phys. Plasmas, 9, 451

Dewar, R. L. 1977, Aust. J. Phys., 30, 533

Fonseca, R. A., Silva, L. O., Tonge, J. W., Mori, W. B., \& Dawson, J. M. 2003, Phys. Plasmas, 10, 1979

Frederiksen, J. T., Hededal, C. B., Haugbølle, T., \& Nordlund, Å. 2004, ApJ, 608, L13

Gruzinov, A. 2001, ApJ, 563, L15

Gruzinov, A., \& Waxman, E. 1999, ApJ, 511, 852

Hededal, C. B., \& Nishikawa, K.-I. 2005, ApJ, 623, L89

Ichimaru, S. 1997, Basic Principles of Plasma Physics, Chap. 4 (W.A. Benjamin Inc., Reading, Ma.), USA

Jackson, J. D. 1975, Classical Electrodynamics, 2nd edn., Chap. 6.5 (New York: John Wiley \& Sons Inc.)

Landau, L. D., \& Lifshitz, E. M. 1975, Classical Theory of Fields, Chap. 10 (Oxford: Pergamon Press Ltd)

Lyubarsky, Y., \& Eichler, D. 2006, ApJ, 647, 1250

Medvedev, M. V., \& Loeb, A. 1999, ApJ, 526, 697

Melrose, D. B. 1973, Plasma Phys., 15, 99

Melrose, D. B. 1980, Plasma Astrophysics (New York: Gordon and Breach Publ.), 1

Melrose, D. B. 2001, Quantum Plasma Dynamics http://www.physics.usyd.edu.au/rcfta/qpd.html

Mészáros, P. 2002, ARA\&A, 40, 137

Mészáros, P. 2006, Rep. Prog. Phys., 69, 2259

Milosavljevic, M., Nakar, E., \& Spitkowsky, A. 2006, ApJ, 637, 765

Piran, T. 2000, Phys. Rep., 333, 529

Piran, T. 2004, Rev. Mod. Phys., 76, 1143

Schaefer-Rolffs, U., Lerche, I., \& Schlickeiser, R. 2006, Phys. Plasmas, 13, 012107

Schlickeiser, R. 2004, Phys. Plasmas, 11, 5532

Silva, L. O., Fonseca, R. A., Tonge, J. W., Mori, W. B., \& Dawson, J. M. 2002, Phys. Plasmas, 9, 2458

Spitkovsky, A., \& Arons, J. A. 2005, ApJ, 603, 669

Stockem, A., Lerche, I., \& Schlickeiser, R. 2006, ApJ, 651, 584

Tautz, R. C., \& Schlickeiser, R. 2005, Phys. Plasmas, 12, 072101

Tautz, R. C., \& Schlickeiser, R. 2006, Phys. Plasmas, 13, 062901

Tautz, R. C., \& Lerche, I. 2006, ApJ, 653, 447

Tautz, R. C., Schlickeiser, R., \& Lerche, I. 2007, J. Math. Phys., 48, 013302

Weibel, E. S. 1959, Phys. Rev. Lett., 2, 83

Wiersma, J., \& Achterberg, A. 2004, A\&A, 428, 365

Yoon, P. H. 2007, Phys. Plasmas, 14, 02454

Yoon, P. H., \& Davidson, R. C. 1987, Phys. Rev. A, 35, 2718 